\title{
LA-UR-19-27257
}

Approved for public release; distribution is unlimited.

Title:

A Verification and Validation Study of the Cyclops I PBX 9502

Experiment A validation of the SURFplus High Explosive Model

Author(s): $\quad$ Grove, John W.

Intended for: Report

Issued: 
Disclaimer:

Los Alamos National Laboratory, an affirmative action/equal opportunity employer, is operated by Triad National Security, LLC for the National Nuclear Security Administration of U.S. Department of Energy under contract 89233218CNA000001. By approving this article, the publisher recognizes that the U.S. Government retains nonexclusive, royalty-free license to publish or reproduce the published form of this contribution, or to allow others to do so, for U.S. Government purposes. Los Alamos National Laboratory requests that the publisher identify this article as work performed under the auspices of the U.S. Department of Energy. Los Alamos National Laboratory strongly supports academic freedom and a researcher's right to publish; as an institution, however, the Laboratory does not endorse the viewpoint of a publication or guarantee its technical correctness. 


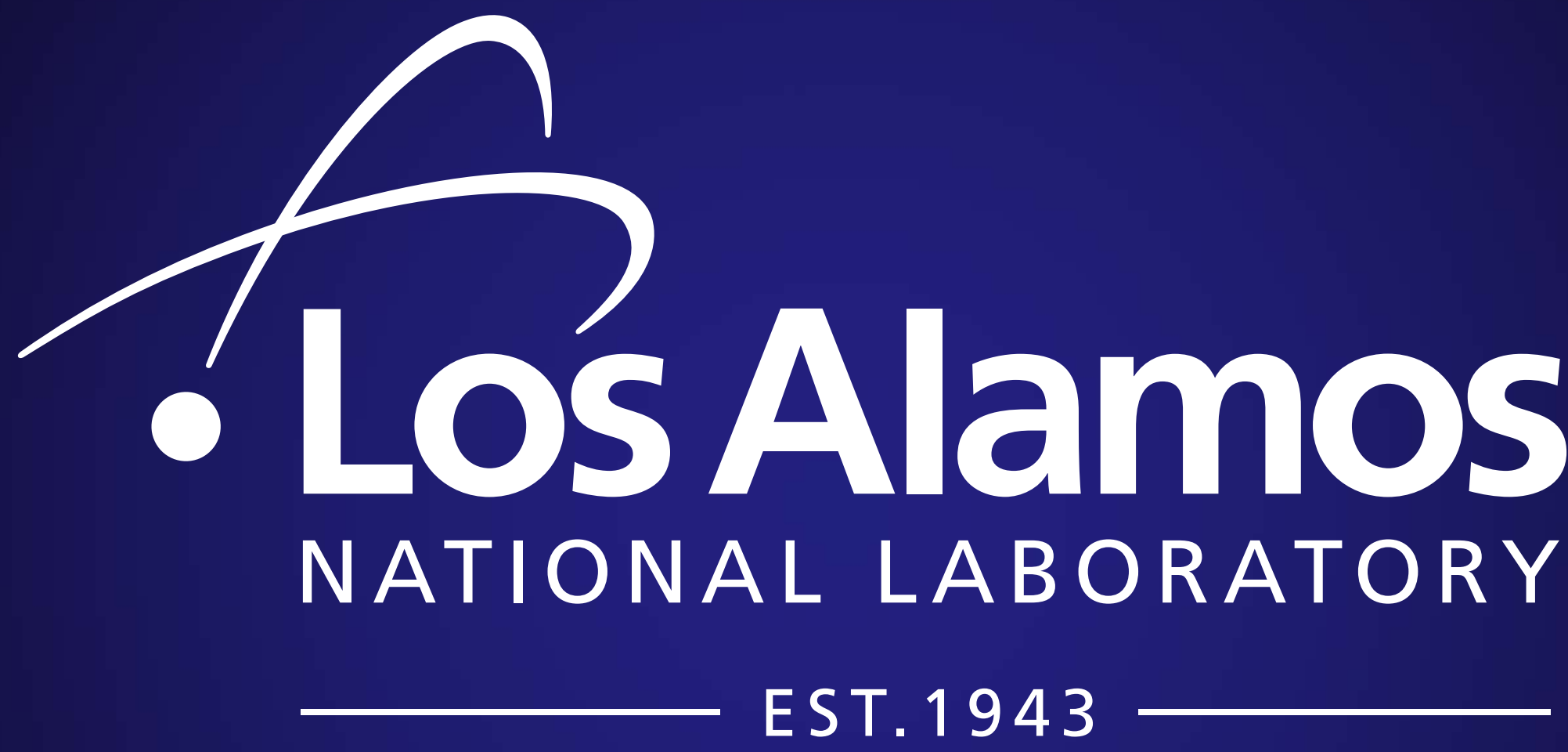




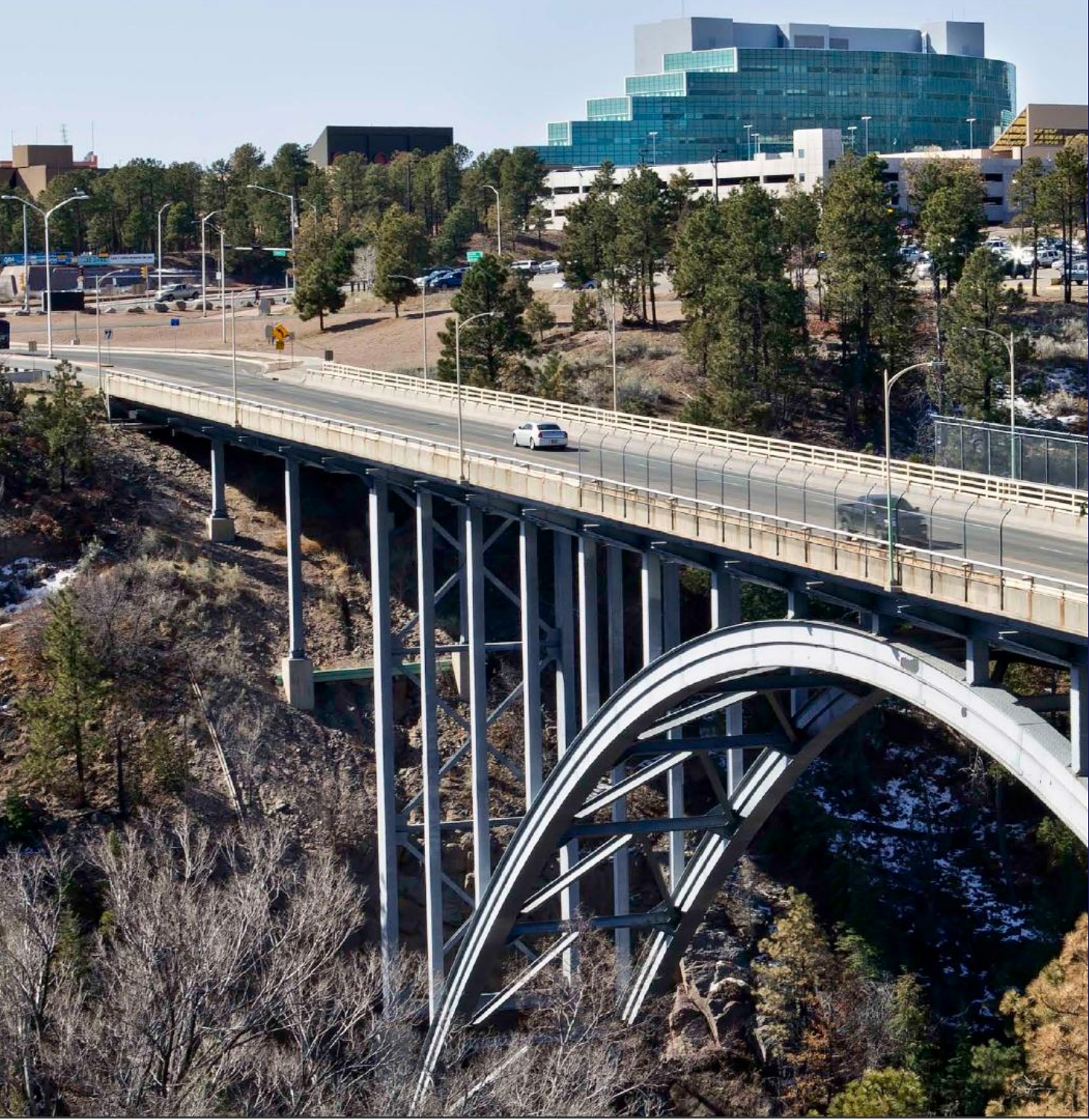

- LosAlamos NATIONAL LABORATORY

EST. 1943

Delivering science and technology

to protect our nation and promote world stability 


\section{A Verification and Validation Study of the Cyclops I PBX 9502 Experiment}

A validation of the SURFplus High Explosive Model

John W. Grove

CCS-2

Tuesday, July 23, 2019 


\section{Contents}

- HE Burn Models

- SURF

- SURFplus

- Programmed Burn

- The Cyclops I experiment

- Simulation Setup

- Results

- Photon-Doppler-Velocimetry Validation

- Proton Radiography Validation

- Conclusions 


\section{Acknowledgements}

- Experimental data

- Guillermo Terrones, Michael W. Burkett, Christopher Morris, David M. Oro

- Pagosa Simulations

- Justin Matthew Hughes, Guillermo Terrones, Erik Frederick Shores

- SURFplus

- Ralph Menikoff

- Discussions and Flag simulations

- Carl Johnson, Adam Coleman

- Michael Steinkamp and Aaron Koskelo suggested this experiment for a validation study and provided the project support for this work

- This work was supported by the ASC Verification and Validation Project's High Explosives Validation effort.

- This work was supported by the US Department of Energy through the Los Alamos National Laboratory. Los Alamos National Laboratory is operated by Triad National Security, LLC, for the National Nuclear Security Administration of U.S. Department of Energy (Contract No. 89233218CNA000001). 


\section{The SURFplus Model}




\section{SURF: Burn initiation}

- SURF is an acronym for Scaled-Unified-Reactive-Front

- Invented by R. Menikoff and S. Shaw

- What follows is a brief synopsis of this model as used for the validation study discussed here. For more details see the xrage user manual and the references cited there.

- SURF and SURFplus are members of a broad category of reactive burn models that use the ignition and growth concept

- The burn front is detected using the Hugoniot function

$$
H=e-e_{0}-\left[\frac{P+P_{0}}{2}\right]\left(V_{0}-V\right)
$$

- The zero subscript corresponds to the state of the undisturbed explosive

- The quantity $H$ is identically zero in this region

- Shock detection is initiated when $H$ becomes positive

- The lead shock is detected when $H$ changes sign or pressure or density decrease

- When a shock is detected in a cell the value $P_{S h}$ is set, which is subsequently advected by the flow $\frac{D P_{s h}}{D t}=0$.

- For more details on the shock detection algorithm see

- R. Menikoff. Shock detector for SURF model. Technical Report LA-UR-16-20116, Los Alamos National Lab., 2016.

URL http://www.osti.gov/scitech/servlets/purl/1234496. 


\section{SURF: Burn propagation}

- Once the shock detector is activated burn is propagated by the equation

$$
\lambda=g(s)
$$

where $\lambda$ is the reaction progress variable (mass fraction of the reaction products), $g(s)$ is a reaction-scale function and $s$ is a dimensionless reaction-scale variable

- The reaction-scale function is given by

$$
\frac{D s}{D t}=f\left(p_{s h}\right)\left\{\begin{array}{cc}
0, & p \leq 0 \\
{\left[\frac{p}{p_{s h}}\right]^{n},} & 0<p<p_{s h} \\
{\left[\frac{p}{p_{s h}}\right]^{n_{h i}},} & p_{s h} \leq p
\end{array}\right.
$$

$$
\begin{gathered}
g(s)=\frac{1}{g_{n}\left(s_{1}\right)}\left\{\begin{array}{cc}
g_{0}(s), & s \leq s_{1} \\
g_{0}\left(s_{1}\right)+g_{0}^{\prime}\left(s_{1}\right)\left(s-s_{1}\right)+\frac{1}{2} g_{0}^{\prime \prime}\left(s_{1}\right)\left(s-s_{1}\right)^{2} & s_{1}<s<s_{\text {cutoff }} \\
g_{n}\left(s_{1}\right) & s>s_{\text {cutoff }} \\
g_{0}(s)=1-e^{-s^{2}} & \\
g_{n}\left(s_{1}\right)=g_{0}\left(s_{1}\right)-\frac{1}{2} \frac{\left[g_{0}^{\prime}\left(s_{1}\right)\right]^{2}}{g_{0}^{\prime \prime}\left(s_{1}\right)}, s_{\text {cutoff }}=s_{1}-g_{0}^{\prime}\left(s_{1}\right) / g_{0}^{\prime \prime}\left(s_{1}\right)
\end{array}\right.
\end{gathered}
$$

- These formulas introduce three dimensionless parameters for the reaction scale evolution equation and the reaction-scale function: $n, n_{h i}$, and $s_{1}$ 


\section{SURF: Shock-Strength Function}

- The shock-strength function in the evolution equation for the reaction scale function used the formula $f\left(p_{\text {sh }}\right)=\frac{\hat{f}\left(\frac{p_{s h}}{p_{\text {scale }}}\right)}{t_{\text {scale }}}$

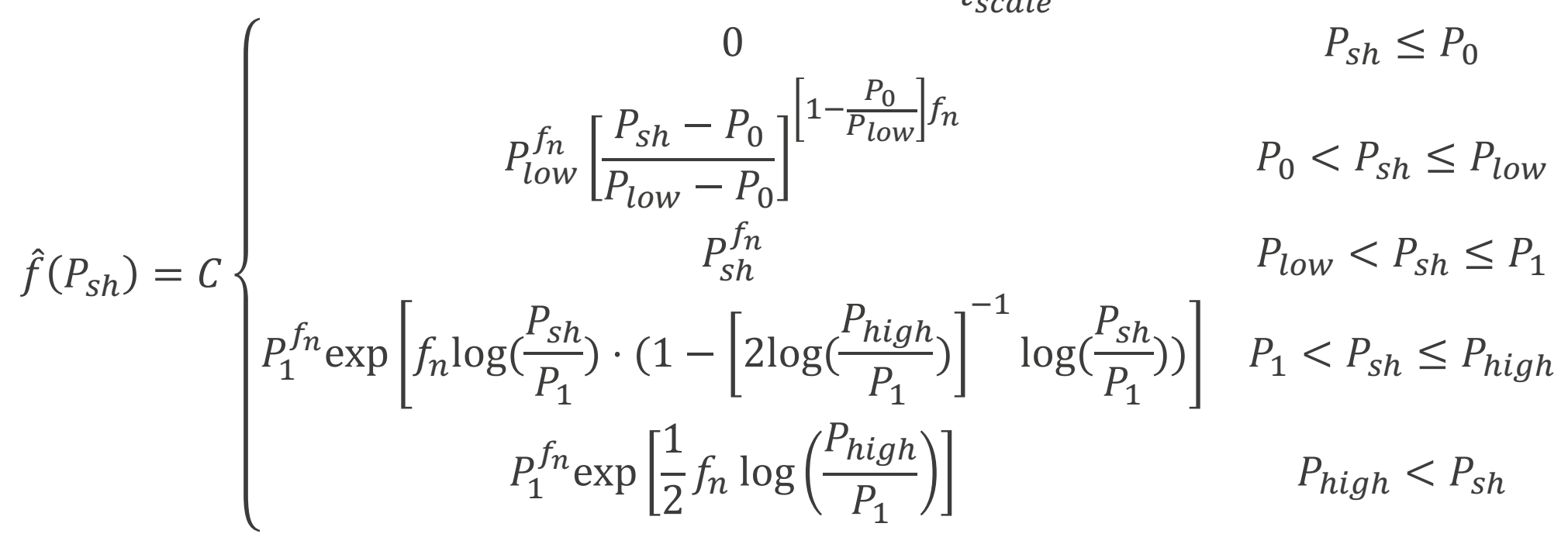

- $f\left(p_{s h}\right)$ is set to zero if the shock detector has not activated unless $p_{s h}>p_{\text {burn }}$ in which case burn will be initiated

- This function is defined by six dimensionless parameters and the pressure scale $C, P_{0}=p_{0} / p_{\text {scale }}, P_{\text {low }}=p_{\text {low }} / p_{\text {scale }}, P_{1}=p_{1} / p_{\text {scale }}, P_{\text {high }}=p_{\text {high }} / p_{\text {scale }}, f_{n}$

- Input for $x$ Rage uses these dimensionless parameters 


\section{SURF: Parameter Values used for PBX-9502}

- The table below summarizes the values for the SURF setup for PBX 9502

\begin{tabular}{|c|c|c|c|c|c|}
\hline Parameter & Value & Parameter & Value & Parameter & Value \\
\hline$t_{\text {scale }}$ & $1 \mu \mathrm{sec}$ & $s_{1}$ & 2.0 & $P_{1}$ & 28.0 \\
\hline$p_{\text {scale }}$ & $1 \mathrm{GPa}$ & $C$ & $4.6 \times 10^{-5}$ & $P_{\text {high }}$ & 60.0 \\
\hline$n$ & 3.2 & $P_{0}$ & 6.0 & $f_{n}$ & 4.05 \\
\hline$n_{h i}$ & 0 & $P_{\text {low }}$ & 8.0 & $p_{\text {burn }}$ & $25 \mathrm{Gpa}$ \\
\hline
\end{tabular}

- For the simulations in this study no addition energy release was added for the SURF model. The reaction energy release was embedded in the products equation of state 


\section{SURF: Model Curves}

- The figures below show the graphs of the reaction-scale function and shock-strength function used by the SURF model. The curves used the parameters from the table on the previous slide.

- The sections of the curves are color coded by region

- Reaction Scale Function: red $s \leq s_{1}$, blue $s_{1}<s \leq s_{\text {cutoff }}$

- Shock-Strength Function: black $\frac{p_{\text {sh }}}{p_{\text {scale }}}<P_{0}$, red $P_{0} \leq \frac{p_{\text {sh }}}{p_{\text {scale }}}<P_{\text {low }}$, green $P_{\text {low }} \leq \frac{p_{\text {sh }}}{p_{\text {scale }}}<P_{1}$, blue $P_{1} \leq \frac{p_{\text {sh }}}{p_{\text {scale }}}<P_{\text {high }}$
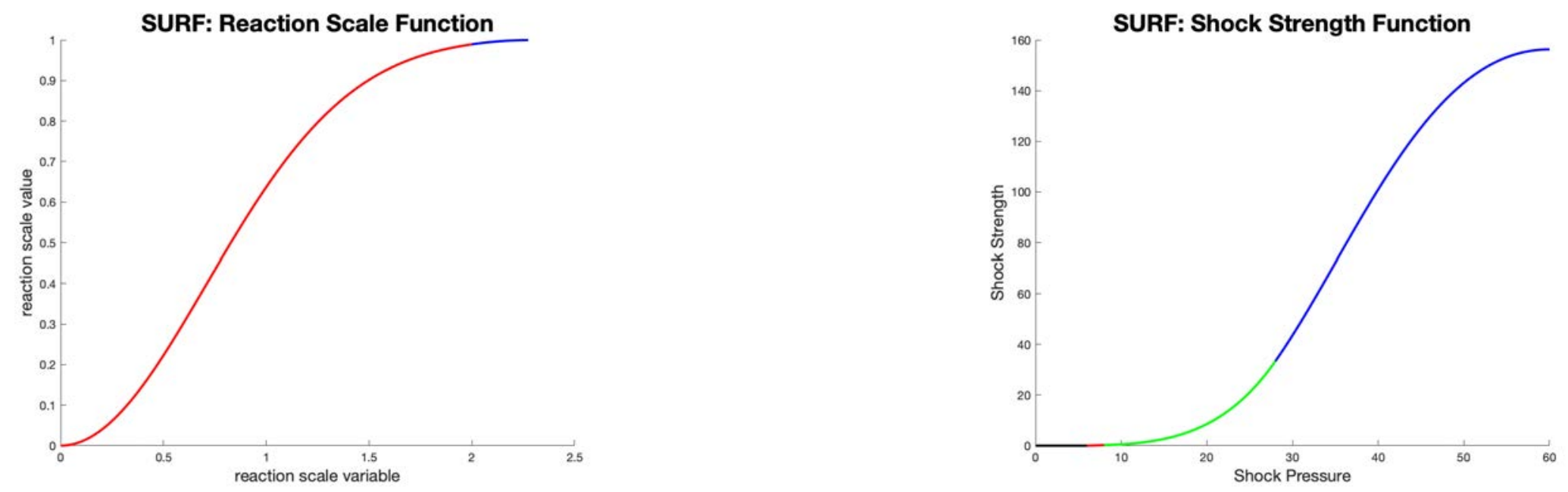


\section{SURFplus}

- SURFplus is an extension of the SURF model that adds a second slower reaction the follows a burn using SURF

- The fast reaction corresponds to molecular decomposition and leads to intermediate products.

- The second reaction burns the intermediate products to produce the final products

- The model system is coupled to the SURF model for the fast reactions via a streamwise ODE and is summarized by the reaction progress variable for intermediate and final products $(\lambda)$ (mass fractions for intermediate and final products) and relative mass fraction of the final products with respect to the intermediate and final products $\left(\lambda_{2}\right)$.

- The energy deficit compared to the equilibrium mixture is modeled as

$$
e_{c c}\left(\lambda_{2}\right)=\left(\left[\frac{1}{1+\left(N_{\text {ratio }}-1\right) \lambda_{2}}\right]^{1 / 3}-N_{\text {ratio }}^{-1 / 3}\right) Q
$$

- Equation of state evaluations use the energy offset

$$
p\left(V, e, \lambda, \lambda_{2}\right)=p\left(V, e-\lambda e_{c c}\left(\lambda_{2}\right), \lambda\right)
$$

Where $p(V, \hat{e} \lambda)$ is the pressure-temperature equilibrium solution for a mixture of reactants and final products 


\section{SURFplus: Evolution equations}

- The evolution equation for $\lambda_{2}$ is modeled as

$$
\frac{D s_{2}}{D t}=\frac{1}{2} \lambda^{2}\left\{\begin{array}{cc}
\frac{h_{1} a_{2}}{h_{1} s_{2}+\left(t_{1} a_{2}-h_{1}\right)\left(h_{1}{ }^{1 / 2}-s_{2}\right)} & s_{2}{ }^{2}<h_{1} \\
a_{2} / s_{2} & h_{1} \leq s_{2}{ }^{2} \leq h_{2} \\
{\left[2\left(1-s_{2}{ }^{2}\right) a_{3}\right]^{1 / 2} / s_{2}} & h_{2}<s_{2}{ }^{2}
\end{array}\right.
$$

- $s_{2}=\sqrt{\lambda_{2}}, a_{2}=\frac{h_{2}-h_{1}}{t_{2}-t_{1}}, a_{3}=\frac{a_{2}{ }^{2}}{2\left(1-h_{2}\right)}$

- $t_{1}, t_{2}, h_{1}, h_{2}$ along with $N_{\text {ratio }}$ and $Q$ are parameters of the model

- For PBX-9502 we used the following values

\begin{tabular}{|c|c|c|c|c|c|}
\hline Parameter & Value & Parameter & Value & Parameter & Value \\
\hline$Q$ & 3 & $t_{1}$ & 0.005 & $t_{2}$ & 0.4 \\
\hline$N_{\text {ratio }}$ & 50 & $h_{1}$ & 0.001 & $h_{2}$ & 0.95 \\
\hline
\end{tabular}

- Note the parameters for time are dimensionless and are actually in units of $t_{\text {scale. }}$. The units for $Q$ should be in $p_{\text {scale }} / \frac{\text { gram }}{c c}$. For the units scaling used here of pressure in gigapascals the specific internal energy units would be kilojoules/gram 


\section{PBX-9501: Programmed Burn Setup}

- The Cyclops I experiment used a PBX-9501 booster to initiate the reaction in the PBX-9502. A detailed high explosive model was not needed for the booster reaction. Since the booster explosive geometry is a simple rectangle the line of sight programmed burn model was sufficient.

- The PBX-9501 burn was started at the location $(6,-0.2)$

- The CJ reactants detonation speed was $D_{C J}=8.8 \mathrm{~km} / \mathrm{sec}$

- The CJ reactants pressure used was $P_{C J}=38.6358 \mathrm{GPa}$.

- The pseudo reaction time used the dimensionless value of 1 .

- In this model, burn is started at location when the spherical wave starting at the initiator location with velocity $D_{C J}$ reaches that point, at which point the pressure is increased to $P_{C J}$ and all reactants are converted into products. In this application the energy release due to the reaction was incorporated into the reaction products equation of state. 


\section{The Cyclops I Experiment}




\section{Experiment Background}

- The Cyclops I high explosive experiment was conducted in 2010 at the Los Alamos National Laboratory to observe the behavior of the insensitive high explosive PBX-9502

- PBX-9502 is a polymer-bonded explosive consisting of $95 \%$ by mass of the insensitive explosive TATB (triaminotrinitrobenzene) and $5 \%$ by mass of the Kel- $F$ (Polychlorotrifluoroethylene) binder

- References:

1. Terrones, G., M.W. Burkett, and C.L. Morris, Burn front and reflected shock wave visualization in an inertially confined detonation of high explosive. AIP Conference Proceedings, 2012. 1426(1): p. 239-242.

2. Hughes, J.M. and G. Terrones, PAGOSA Simulations for the LANL Blast Tube, Los Alamos National Laboratory, LA-UR-18-27325. 2018.

3. Hughes, J.M., G. Terrones, and E.F. Shores, Cyclops I: Modeling Error Study for Inertially Confined Detonation of Insensitive High Explosive (IHE) PBX-9502, Los Alamos National Laboratory, LA-UR-17-28074. 2017.

4. Terrones, G., Cyclops Proof Shot, Los Alamos National Laboratory, LA-UR-10-03963. 2010.

5. Terrones, G., M.W. Burkett, and C. Morris, Burn Front Data from the Cyclops I Experiment, LA-UR-11-07121. 2011.

6. Terrones, G., M.W. Burkett, and C. Morris, Burn Front and Reflected Shock Wave Visualization in an Inertially Confined Detonation of High Explosive, Los Alamos National Laboratory, LA-UR 11-03382. 2011.

7. Terrones, G., et al., Cyclops Experiment, Los Alamos National Laboratory, LA-UR-1004469. 2010. 


\section{Experimental Device}

- The device consisted of a steel cylinder containing PBX 9502 with an inner shell of tin in the form of an ellipse whose major axis is rotated relative to the detonator.

- A photograph of the apparatus taken from reference 1 is shown on the right.

- The experiment was diagnosed with a set of eight Photon-Doppler-Velocimetry (PDV) probes on the outside of the device and proton radiography images of the explosion at one microsecond intervals.

- A schematic of the device showing dimensions is shown on the right.

- The PBX-9502 was ignited using a line wave generator (LWG) to ignite a PBX9501 booster that penetrates the steel shell.

Steel 304L Cylindrical Shell
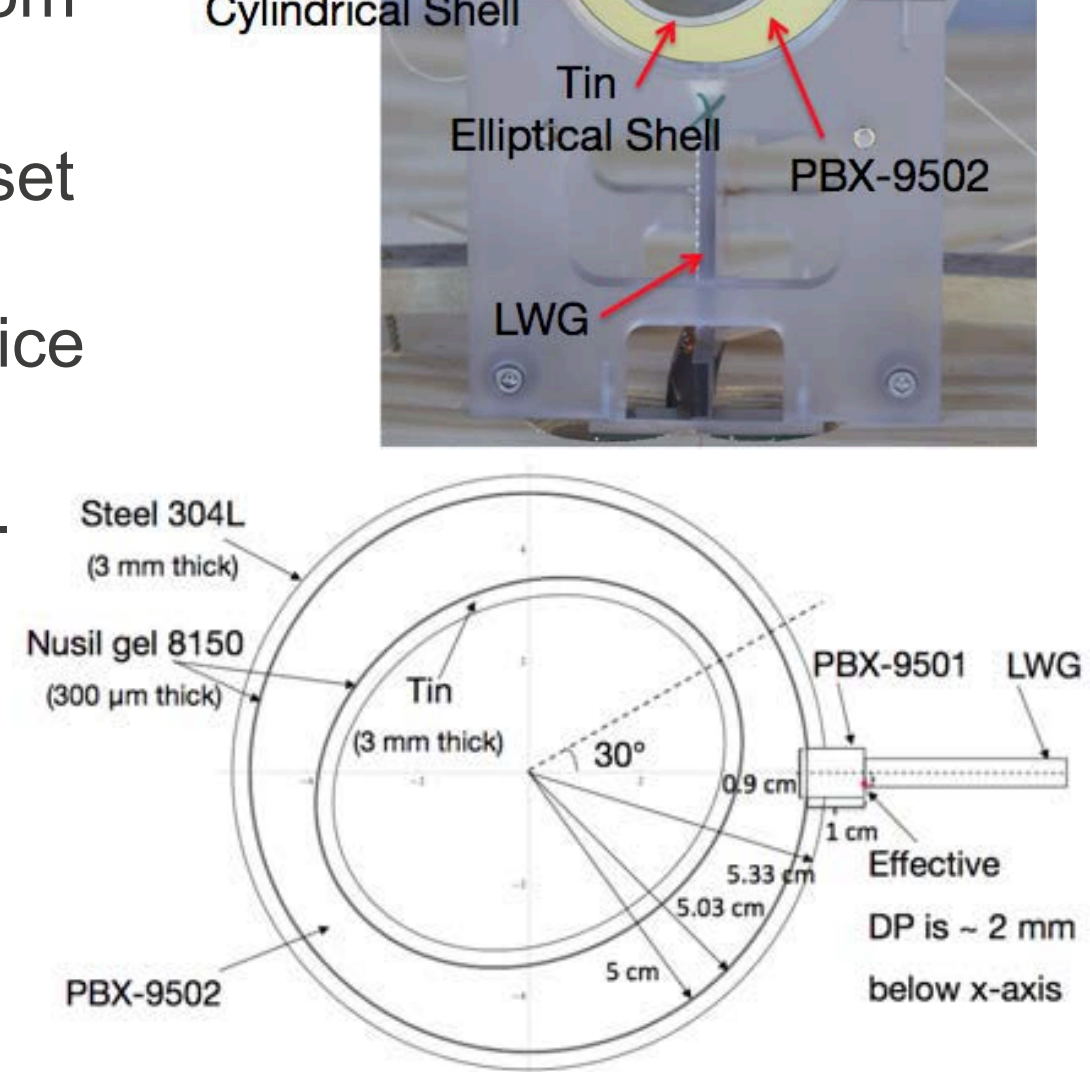


\section{Simulation Setup}




\section{Background}

- This experiment was simulated using the Los Alamos Eulerian Applications Code xRage

- xRage is a fixed grid Eulerian code with adaptive cell-by-cell mesh refinement

- The flow model is the multi-material inviscid Euler equations

- Mixed cells are treated using the Amagat material separated equation of state model with pressure-temperature equilibrium

- The high explosives are modeled using the SURFplus reactive burn models coupled to the xRage Eulerian solver

1. Menikoff, R. and M.S. Shaw. Reactive burn models and ignition \& growth concept. in EPJ Web Confs. 10. 2010. EDP Sciences.

2. Shaw, M.S. and R. Menikoff. A reactive burn model for shock initiation in a PBX: scaling and separability based on the hot spot concept. in Fourteenth International Symposium on Detonation. 2010. Coeur d'Alene, Idaho, USA: US office of naval research.

3. Menikoff, R. and M.S. Shaw, The SURF model and the curvature effect for PBX 9502. Combustion Theory and Modelling, 2012. 16(6): p. 1140-1169.

4. Menikoff, R., Shock Detector for SURF Model, Los Alamos National Laboratory, LA-UR-16-20116. 2016.

5. Menikoff, R., SURFplus Model Calibration for PBX 9502, Los Alamos National Laboratory, LA-UR-17-31015. 2017. 


\section{Simulation Setup}

- The computational domain extends from $-7.5 \mathrm{~cm} \leq x \leq 17.5 \mathrm{~cm}$ and $-7.5 \mathrm{~cm} \leq y \leq 7.5 \mathrm{~cm}$ in standard Cartesian geometry.

- The base computational mesh used square grid blocks of size 200 microns $=.02 \mathrm{~cm}$.

- The AMR specification for the various materials are described in the following table

\begin{tabular}{|c|c|c|c|c|c|c|c|}
\hline $\begin{array}{c}\text { PBX 9502 } \\
\text { Burn Zone } \\
\text { Size }\end{array}$ & $\begin{array}{l}\text { PBX 9501 } \\
\text { Burn Zone } \\
\text { Size }\end{array}$ & Air & $\begin{array}{c}\text { PBX 9501 } \\
\text { reactants } \\
\text { and } \\
\text { products }\end{array}$ & $\begin{array}{c}\text { PBX 9502 } \\
\text { reactants and } \\
\text { products }\end{array}$ & Sylgard & $\begin{array}{c}\text { Stainless } \\
\text { Steel SS-304L }\end{array}$ & Tin \\
\hline $\mathbf{2 0 0}$ & 200 & 200 & 200 & 200 & 100 & 100 & 100 \\
\hline $\mathbf{1 0 0}$ & 100 & 100 & 100 & 100 & 100 & 100 & 100 \\
\hline $\mathbf{5 0}$ & 50 & 100 & 50 & 50 & 50 & 50 & 50 \\
\hline $\mathbf{2 5}$ & 50 & 100 & 50 & 25 & 50 & 50 & 50 \\
\hline $\mathbf{1 2 5}$ & 200 & 200 & 200 & 12.5 & 200 & 25 & 25 \\
\hline $\mathbf{6 . 2 5}$ & 200 & 200 & 200 & 12.5 & 200 & 25 & 25 \\
\hline
\end{tabular}

- The experiment used Nusil gel 8150 to bind the PBX-9502 to the metal shells, but due to lack of equation of state data for this material we substituted the Nusil gel with the Sesame material for Sylgard.

- Both materials are silicon based elastomers and should have similar hydrodynamic properties. 


\section{Initial Conditions}

- The figure shows the initial geometry used for the Cyclops I setup

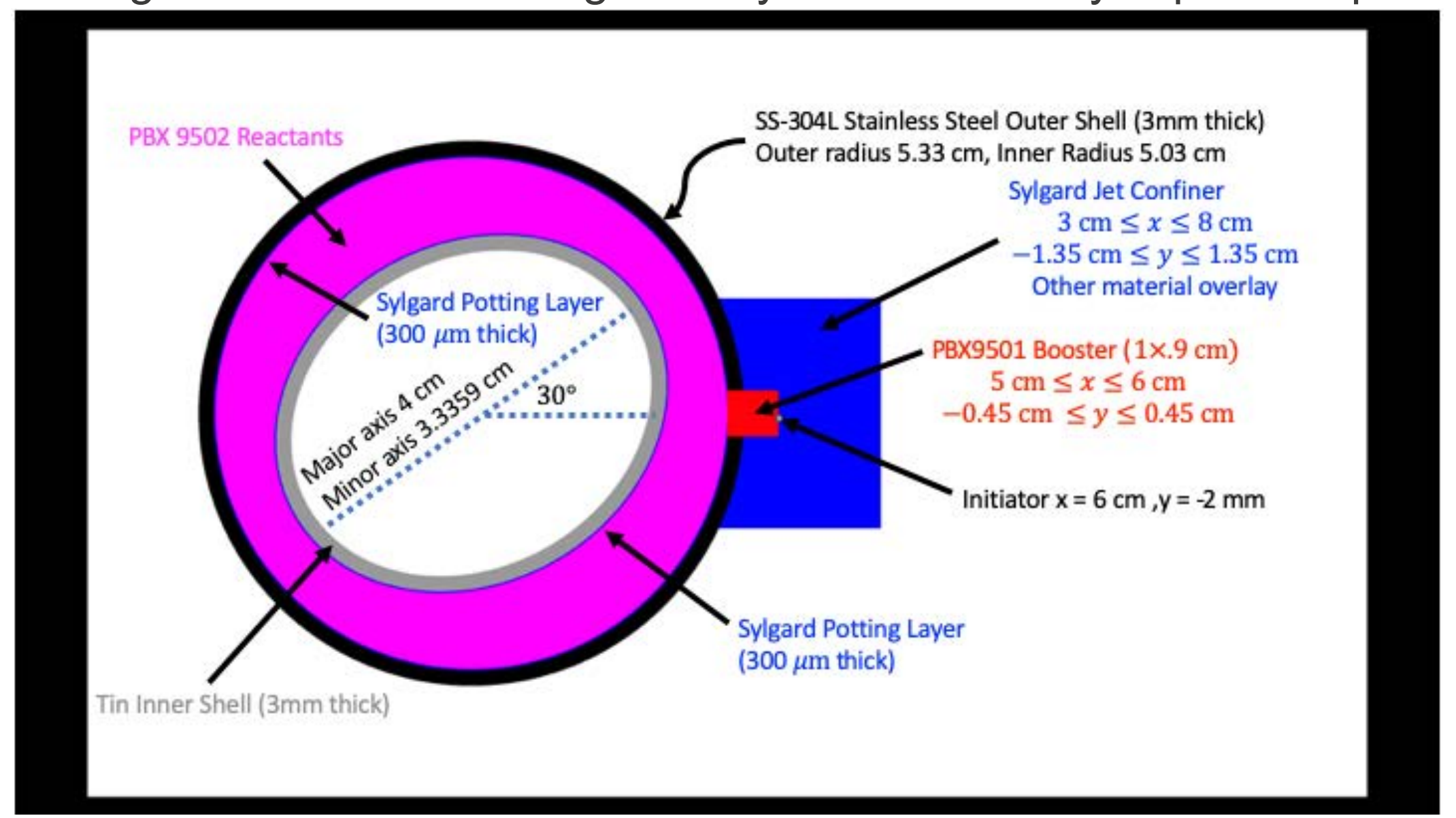




\section{Initial Conditions: Jet Confiner}

- The main difference in the computational setup and the experiment was the addition of a block of plastic around the PBX-9501 booster.

- This block was needed to suppress mixing between the ejected PBX-9501 products and the background air. Without this box xRage gets a time step crash due to mixing between these two materials.

- The box has no effect on the propagation of the PBX-9502 burn front in the interior of the device. It just helps avoid a time step crash unrelated to the HE burn model.

- A illustration of this jet is shown on the right.

- Note that for a Lagrangian simulation this jet would be a source of grid tangling.

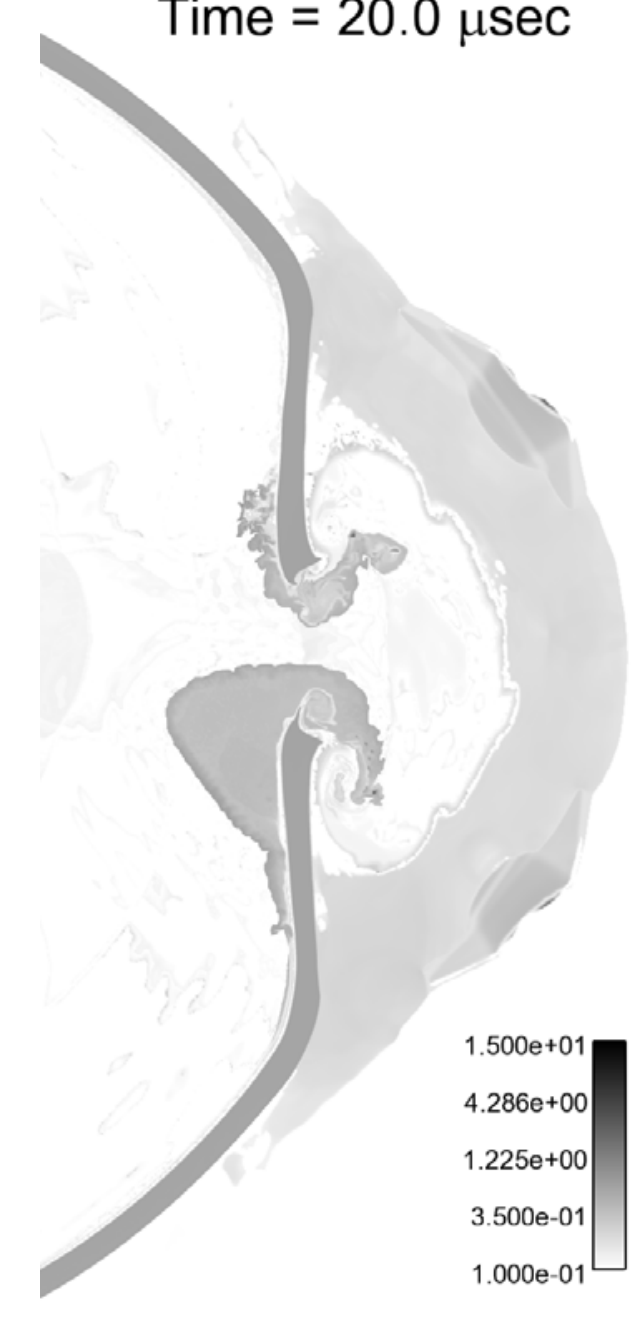




\section{Initial Conditions: Flow Cauchy Data}

- The table below summarizes the initial conditions used for these simulations. All materials are initially at rest.

\begin{tabular}{|c|c|c|c|c|}
\hline Material & $\begin{array}{c}\text { Pressure } \\
\text { (Mbar) }\end{array}$ & Temperature (K) & $\begin{array}{c}\text { Density } \\
\text { (grams/cc) }\end{array}$ & $\begin{array}{c}\text { Specific Internal } \\
\text { Energy (tera } \\
\text { erg/gram) }\end{array}$ \\
\hline Air & $1.0 \times 10^{-6}$ & 296.15 & 0.001 & $2.5 \times 10^{-3}$ \\
\hline SS-304L Steel & $1.0 \times 10^{-6}$ & 296.15 & 7.89 & $8.48 \times 10^{-5}$ \\
\hline Tin & $1.0 \times 10^{-6}$ & 296.15 & 7.27 & $2.53 \times 10^{-5}$ \\
\hline Sylgard & $1.0 \times 10^{-6}$ & 296.15 & 1.04 & $-2.8 \times 10^{-5}$ \\
\hline $\begin{array}{c}\text { PBX 9501 } \\
\text { reactants }\end{array}$ & $1.0 \times 10^{-6}$ & 296.15 & 1.894 & $-3.61 \times 10^{-5}$ \\
\hline $\begin{array}{c}\text { PBX 9502 } \\
\text { reactants }\end{array}$ & $1.0 \times 10^{-6}$ & 296.15 & 1.84 & $-4.43 \times 10^{-5}$ \\
\hline
\end{tabular}

- The PBX-9501 was initiated using a program burn for a point on the right hand side of the booster $2 \mathrm{~mm}$ below the $\mathrm{x}$-axis. 


\section{Hydrodynamic Equation of State Models}

- The background material for the simulation is air modeled as a perfect gas with Grüneisen coefficient $\Gamma=\gamma-1=0.4$ and a specific heat at constant volume of $C_{V}=8.4417 \times 10^{-6} \frac{\text { Tergs }}{\text { gram } K}$.

- The high explosive reactants and products used Sesame equations of state.

\begin{tabular}{|c|c|c|c|c|c|}
\hline Material & Sesame ID & Material & Sesame ID & Material & Sesame ID \\
\hline PBX 9501 Reactants & 950104 & PBX 9502 Reactants & 950202 & Sylgard \\
\hline PBX 9501 Products & 8211 & PBX 9502 Products & 950211 & \\
\hline
\end{tabular}

- The metal shells were modeled using the Steinberg-Mie-Grüneisen equation of state with constant specific heats at constant volume

$$
\begin{gathered}
P-P_{r}(V)=\frac{\Gamma(V)}{V}\left(e-e_{r}(V)\right)=\frac{\Gamma(V)}{V} C_{V}\left(T-T_{r}(V)\right) \\
C_{V}\left\{T_{r}^{\prime}(V)+\frac{\Gamma(V)}{V} T_{r}(V)\right\}=e_{r}(V)+P_{r}^{\prime}(V)
\end{gathered}
$$

- For compression the reference pressure and specific volume assume a linear relation between the shock velocity $\left(U_{S}\right)$ and particle velocity behind the shock $\left(U_{P}\right)$ at constant compression $U_{S}=c_{0}+s U_{P}$ where $\mathrm{s}$ is a quadratic function of the compression $\left(V_{0}-V\right) / V_{0}$

- For expansion the stiff gamma law equation of state is used. 


\section{Steinberg Equation of State Parameters}

- We used the equation of state parameters for SS-304L stainless steel and tin taken from the report

- Steinberg, D.J., Equation of State and Strength Properties of Selected Materials, Lawrence Livermore National Laboratory, UCRL-MA-106439-rev. 1996.

- The table below summarized the values for the Steinberg-MieGrüneisen equation of state used for this study.

\begin{tabular}{|c|c|c|}
\hline Material & Steel (SS-304L) & Tin \\
\hline Reference density $\left(\rho_{0}\right)$ grams/cc & 7.9 & 7.287 \\
\hline Grüneisen exponent at reference density $\left(\Gamma_{0}\right)$ & 1.93 & 2.27 \\
\hline Grüneisen exponent at infinite compression (b) & 0.5 & 0.54 \\
\hline Reference sound speed $\left(c_{0}\right) \mathrm{cm} / \mu \mathrm{sec}$ & 0.457 & 0.259 \\
\hline$S_{1}$ & 1.49 & 1.49 \\
\hline$S_{2}$ & 0 & 0 \\
\hline$S_{2}$ & 0 & 0 \\
\hline Specific heat at constant volume $\left(C_{V}\right) \frac{\text { Teraerg }}{\operatorname{aram}} /{ }^{\circ} \mathrm{K}$ & $1.2417 \times 10^{-4}$ & $3.051 \times 10^{-5}$ \\
\hline
\end{tabular}




\section{Material Strength Models}

- Both the steel and tin shells that bound the PBX-9502 were modeled using the Steinberg-Cochran Guinan hypoelastic-plastic flow model.

- Steinberg, D., S. Cochran, and M. Guinan, A Constitutive Model for Metals Applicable at High Strain-Rate. J. Appl. Phys., 1980. 51: p. 1498-1504.

- The material parameters used are summarized in the table below.

\section{Material}

Shear Modulus, $\left(G_{0}\right)$ Mbar

Yield Strength, $\left(Y_{0}\right)$ Mbar

Pressure dependence for the shear modulus (A) 1/

Mbar

Temperature dependence for the shear modulus (B) $1 /{ }^{\circ} \mathrm{K}$

Work hardening parameter $(\beta)$ Mbar

Initial plastic strain

Work hardening maximum $\left(Y_{\max }^{*}\right)$ Mbar

Coefficient of volume dependence of the Grüneisen exponent

Melt $\left(\mathrm{T}_{\mathrm{mo}}\right)^{\circ} \boldsymbol{K}$

\begin{tabular}{|c|c|}
\hline Stainless Steel SS-304L & Tin \\
\hline 0.770 & 0.179 \\
$3.4 \times 10^{-3}$ & $1.6 \times 10^{-3}$ \\
2.26 & 8.66 \\
\hline $.55 \times 10^{-4}$ & $2.12 \times 10^{-3}$ \\
43.0 & 2000.0 \\
0 & 0 \\
$2.5 \times 10^{-3}$ & $2.2 \times 10^{-3}$ \\
$\Gamma_{0}-b$ & $\Gamma_{0}-b$ \\
2380 & 656.6 \\
\hline
\end{tabular}




\section{Photon-Doppler-Velocimetry Validation}




\section{PDV Validation}

- When the burn front shock wave interacts with the bounding metal shells, transmitted waves are created which reverberate inside the shells and transmit waves into the bounding materials. When this lead burn front shock passes near a PDV location in the steel shell these waves propagate through the shell and are picked up by the PDV probes as motion of the outer steel shell.

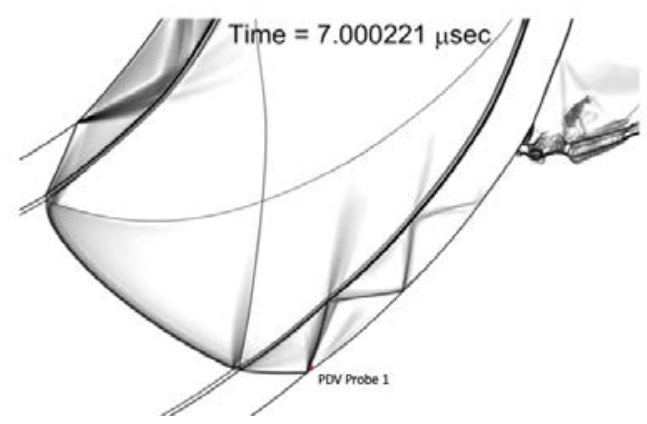

- The arrival times for the burn fronts at the PDV locations were tracked using Lagrangian tracers

- Currently xRage does not support true PDV probes in Cartesian geometry

- The main difference between Lagrangian tracers and PDV probe tracers is that PDV probes are constrained to only move in the direction of the PDV probe beam.

- This difference means that the Lagrangian probes will move away from the probe beam with time resulting in an effectively higher velocity since they stay closer to the lead burn wave than a true PDV probe.

- In our case the Lagrangian tracers are sufficient to get the first arrival time at the probe location. 


\section{PDV Probe Locations}

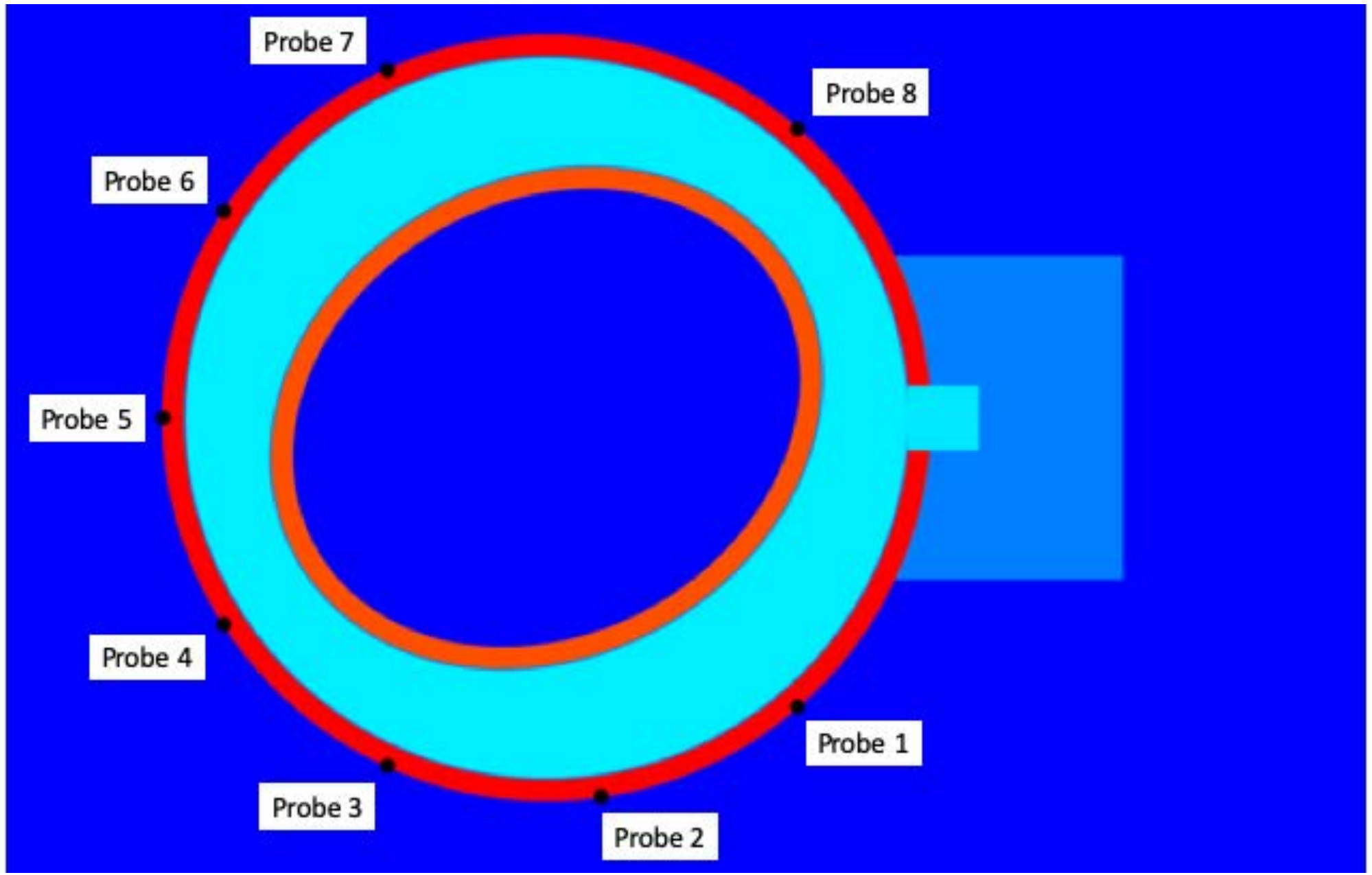




\section{Convergence Results for Shock Arrival Times}

- We performed a convergence analysis for the time of first arrival of the burn front at the eight PDV probe locations.

- The velocity at the Lagrangian Tracers was extracted from the text file $x$ Rage prints for the tracer data and processed using a MATLAB script.

- The experiment did not record a starting time. This value was estimated by fitting the experimental data to align with the first PDV probe computed arrival.

- A linear regression analysis shows that the first arrival times at the probes converge to experiment at approximately first order.

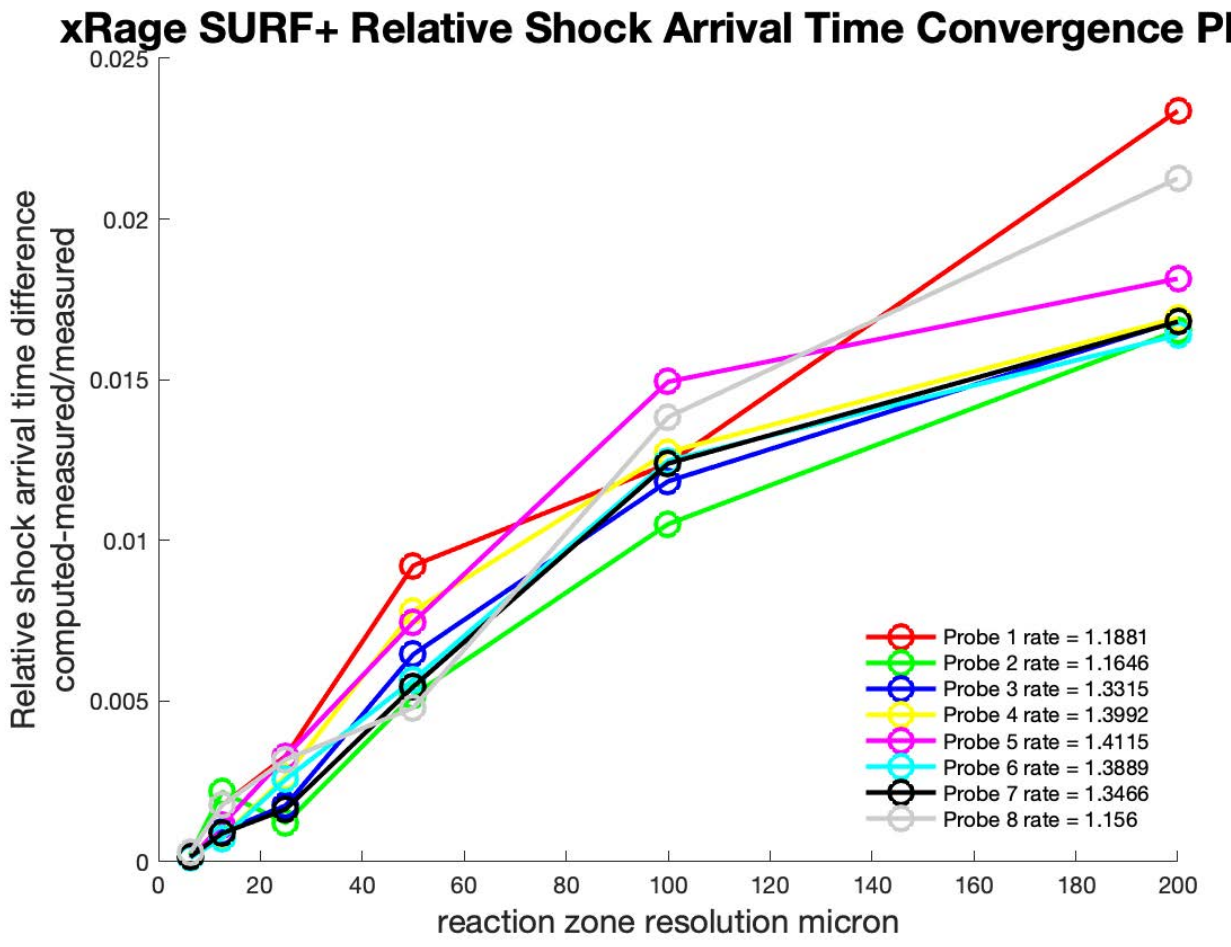




\section{Arrival Time Convergence}

- At low resolutions all of the probes show a lag in the first arrival time of the burn front

- At higher resolutions this lag largely disappears

- By the 6.5 micron case the arrival times are very close to the experimental values

- The arrival time for the experimental data was set by locating the first sharp rise in the PDV data

- Computational arrival times were found by locating the time of the pressure maximum at the Lagrangian tracers.

- Probe 5 is somewhat different from the other probes since at the time of first arrival it is experiencing two burn front accelerations from either side of the device.

- The first jump for the experiment line up will with the higher resolution data

- The second jump comes about 3 microseconds after the first jump and is not readily apparent in the computations. 


\section{PDV Probes 1-3 Arrival Times}

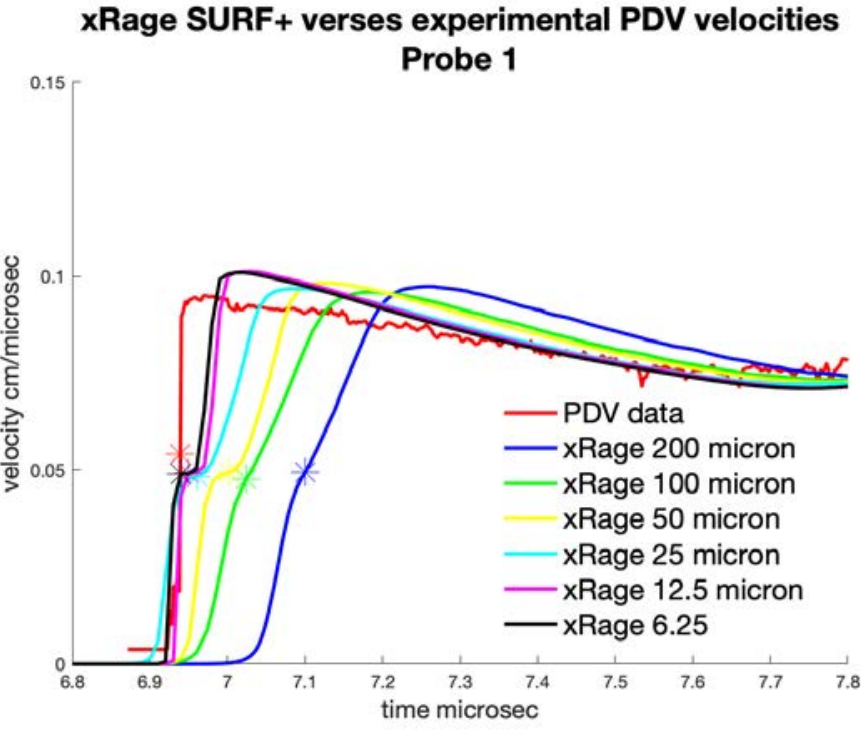

xRage SURF+ verses experimental PDV velocities

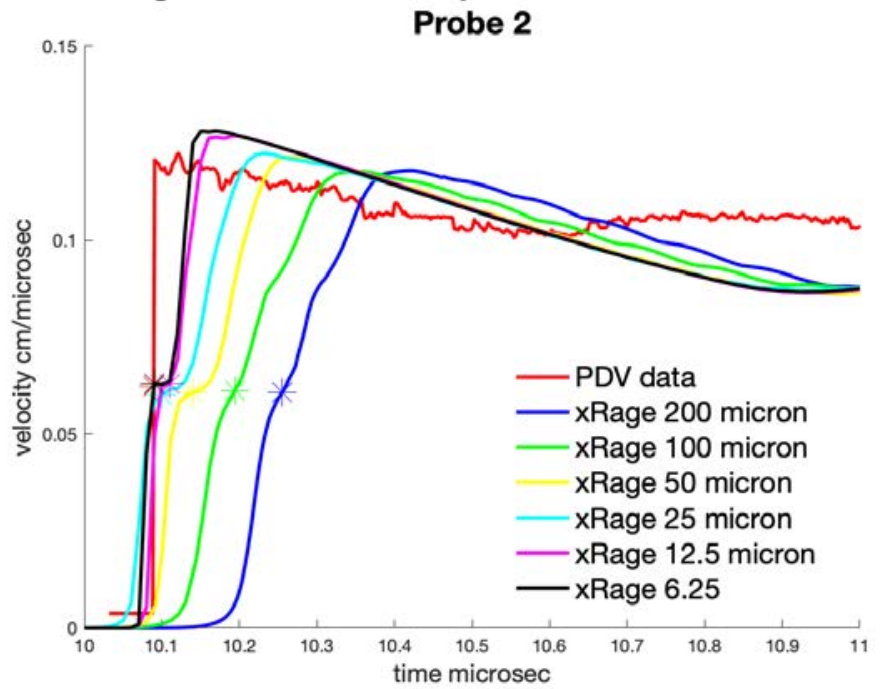

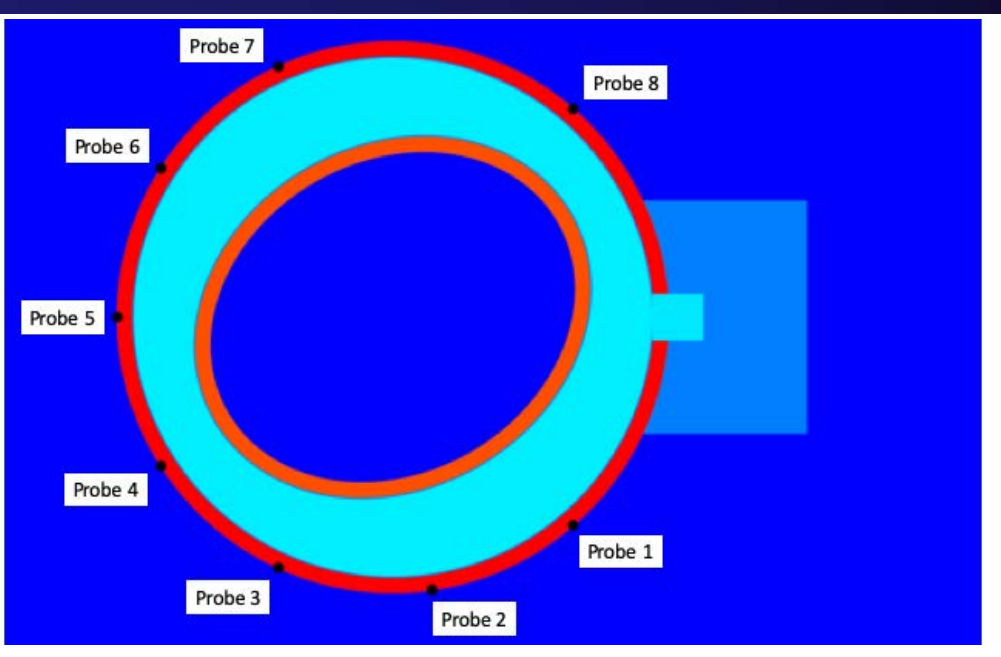

xRage SURF+ verses experimental PDV velocities Probe 3

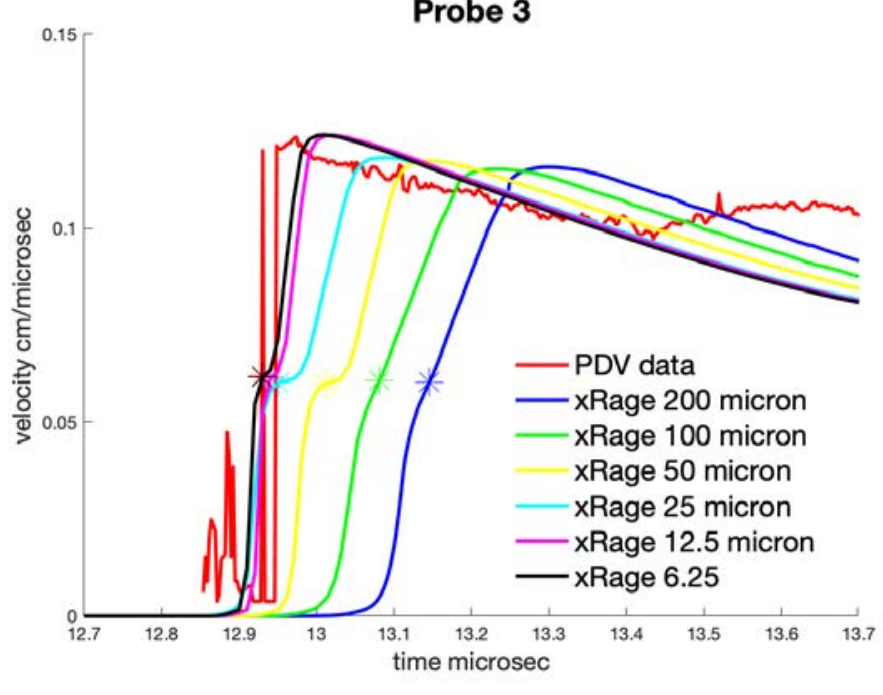




\section{PDV Probes 4-6 Arrival Times}

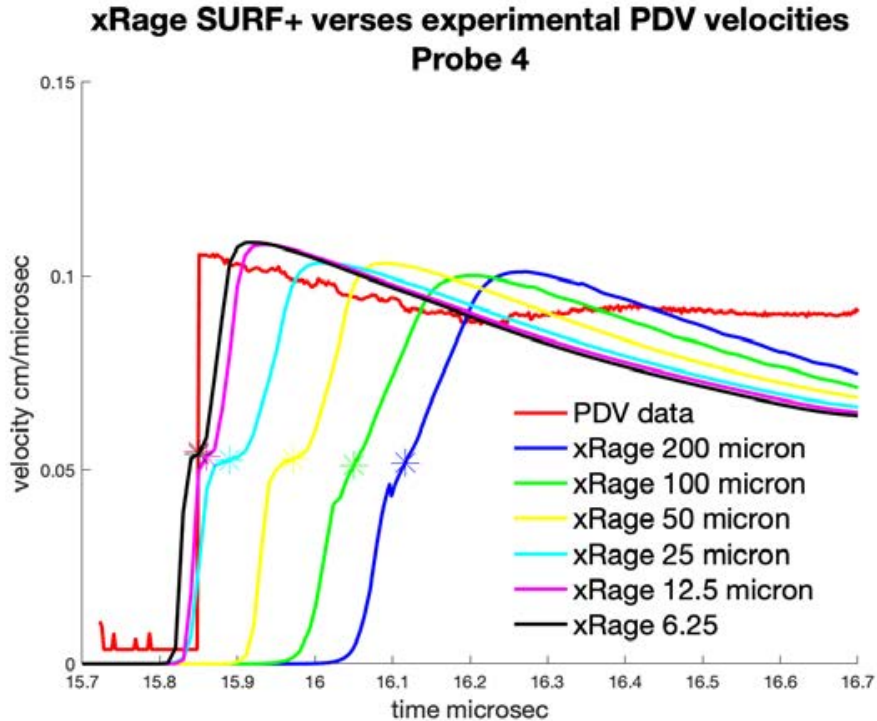

XRage SURF+ verses experimental PDV velocities

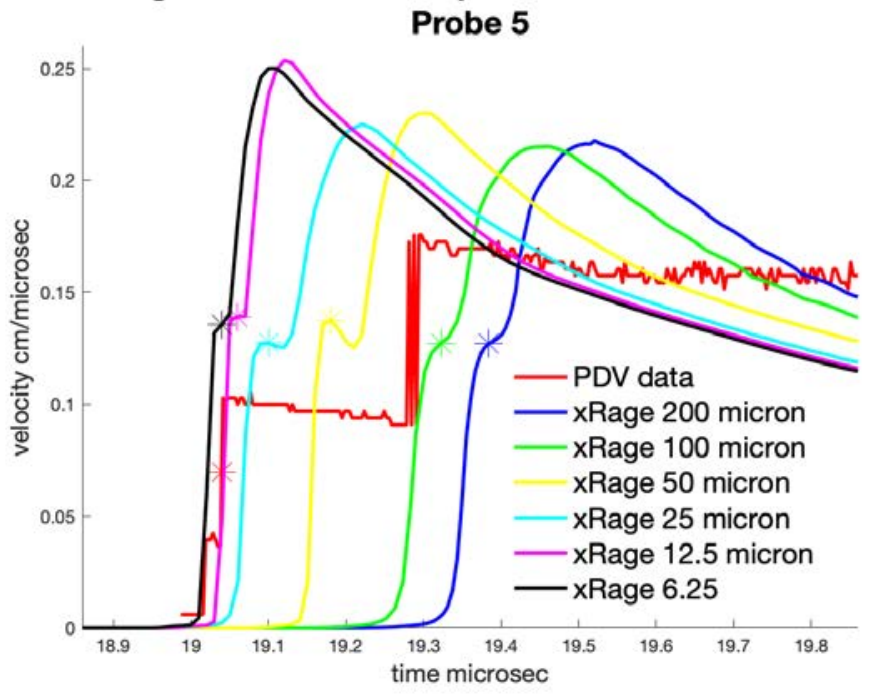

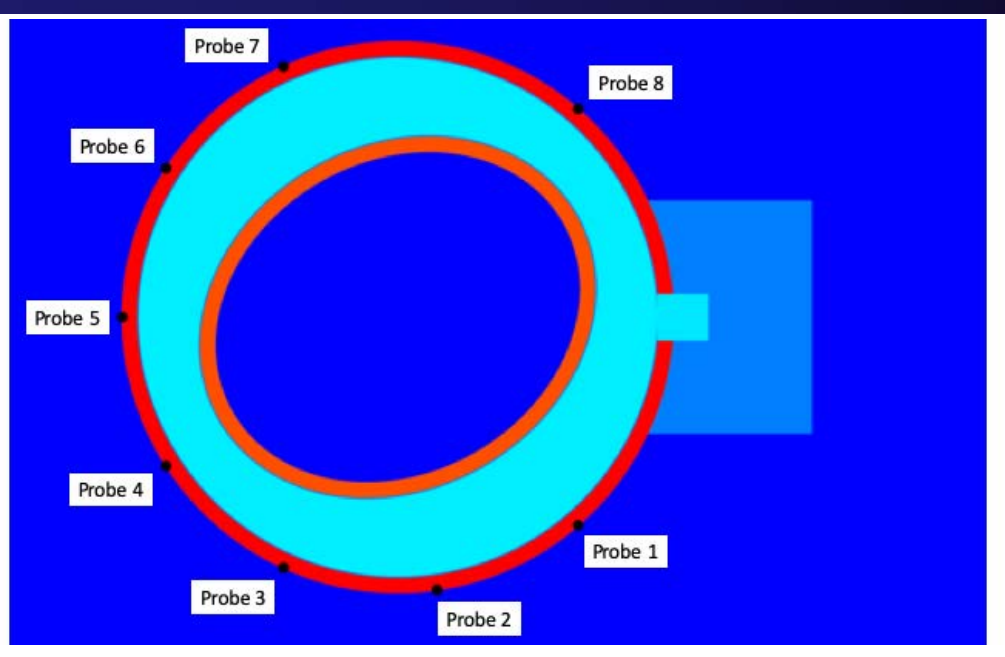

xRage SURF+ verses experimental PDV velocities Probe 6

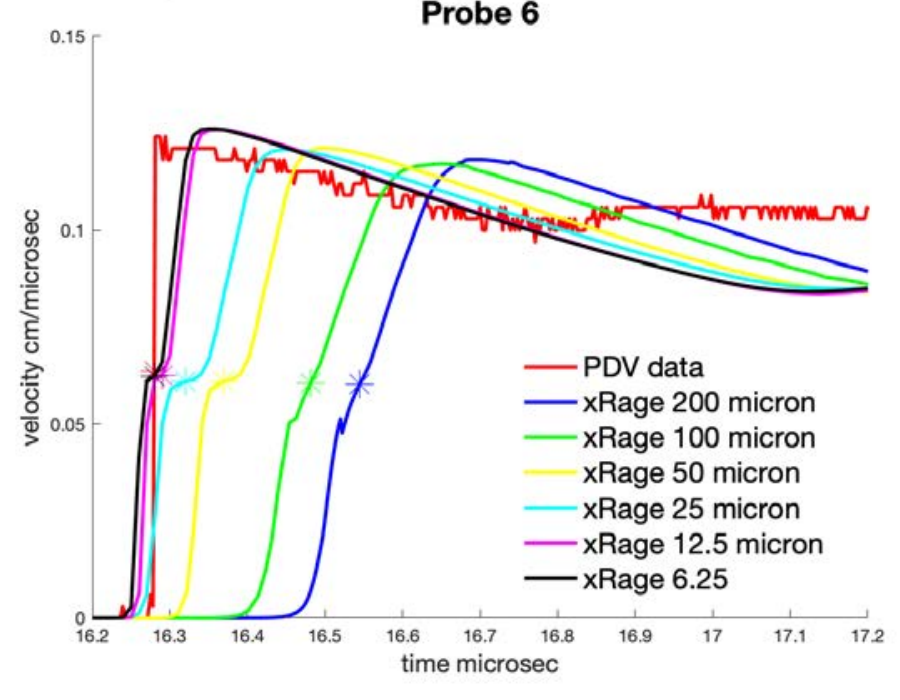




\section{PDV Probes 7 and 8 Arrival Times}
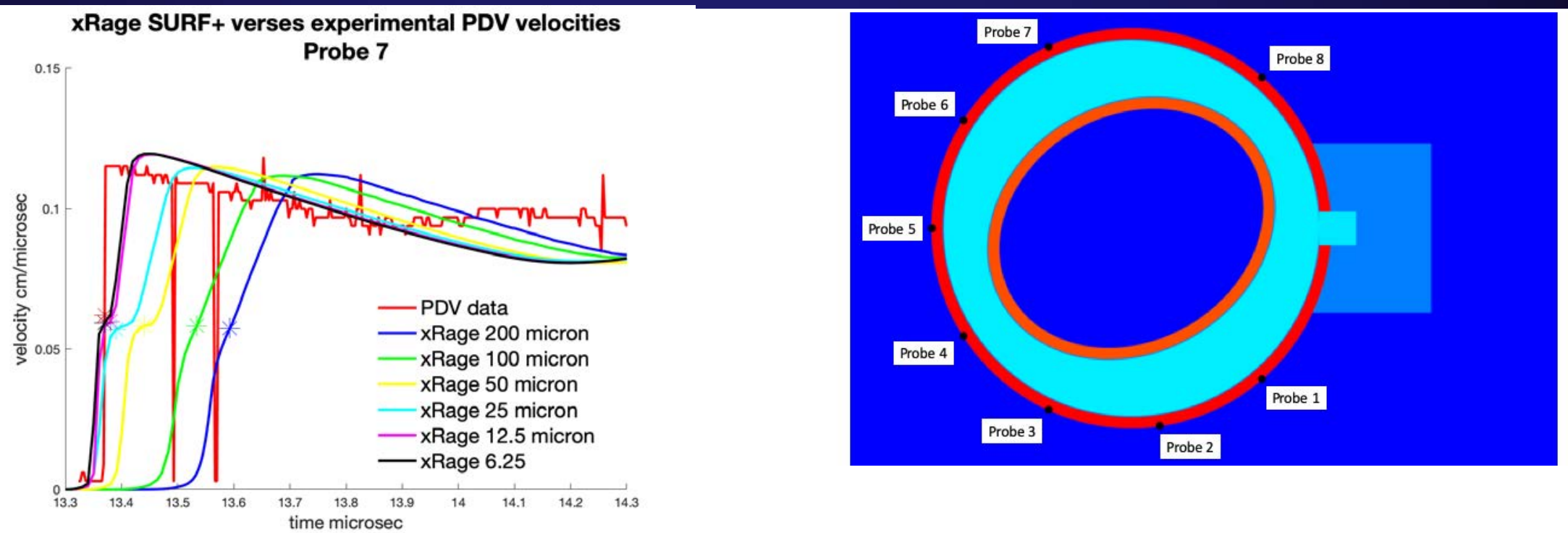

xRage SURF+ verses experimental PDV velocities

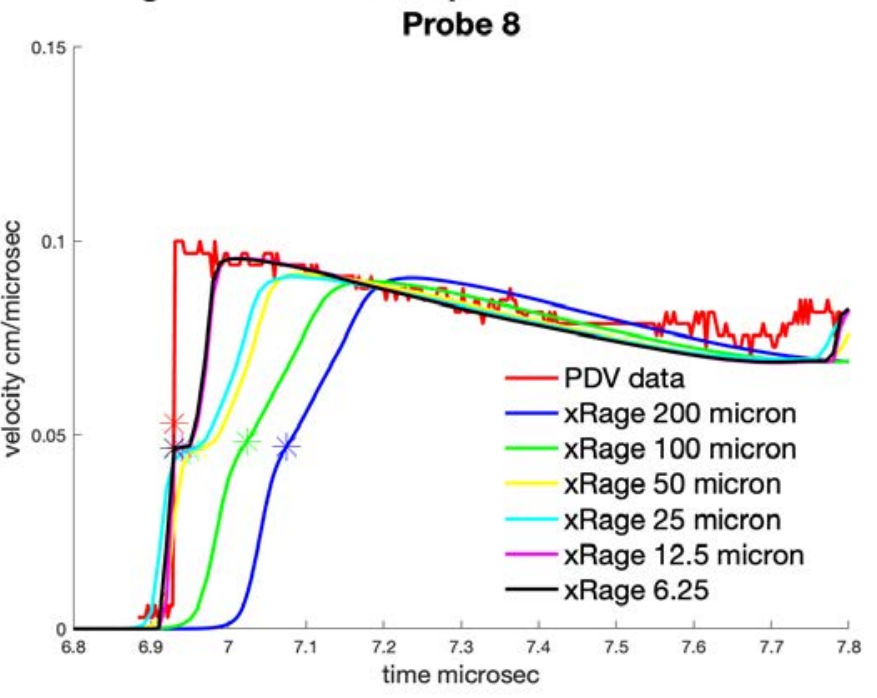




\section{Verification Results}

- We use the 6.5 micron as a pseudo fiducial solution and compute the rates of convergence of the coarser resolutions to the fiducial for the finest arrival time (peak pressure for the probe data)

- The arrival times show first order convergence

- The velocity at time of first arrival is nearly constant and does not show any significant convergence behavior

- Pressure shows a considerable spread at low resolution and appears to be nearly constant at higher resolutions
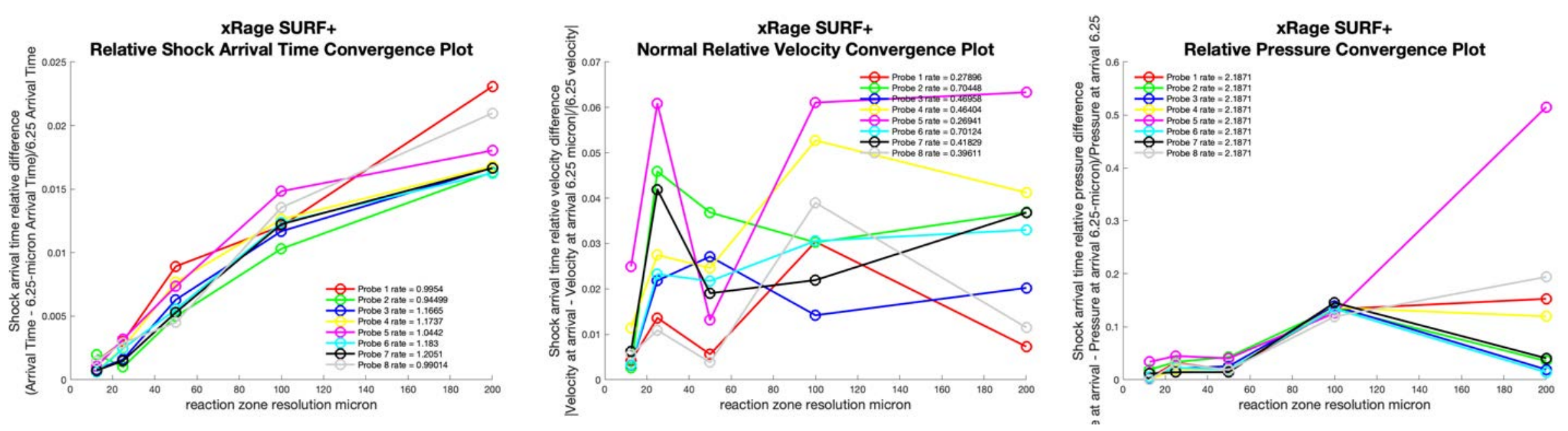


\section{Proton Radiography Validation}




\section{Proton Radiography Images}

- The Cyclops I experiment was imaged using proton radiography with data in the form of an audio video interleave (AVI) movie generated from the PRAD photographs.

- The individual frames of this movie were extracted using MATLAB image processing tools in the form of nineteen $1161 \times 1161$ pixel raster files.

- These raster files were used to create EnsightGold format files that could be overlaid with the computed solutions.

- Ensight provided a number of useful functions that included

- Panning and zooming

- Modification of color maps

- Ability to easily move forward on backward in time over the images

- Adding annotations

- It was determined that the images were taken at one microsecond intervals starting four microseconds after booster ignition. 


\section{One Movie Shows it All}

$$
200 \mu \mathrm{m} \quad \text { Time }=0.0 \mu \mathrm{sec}
$$

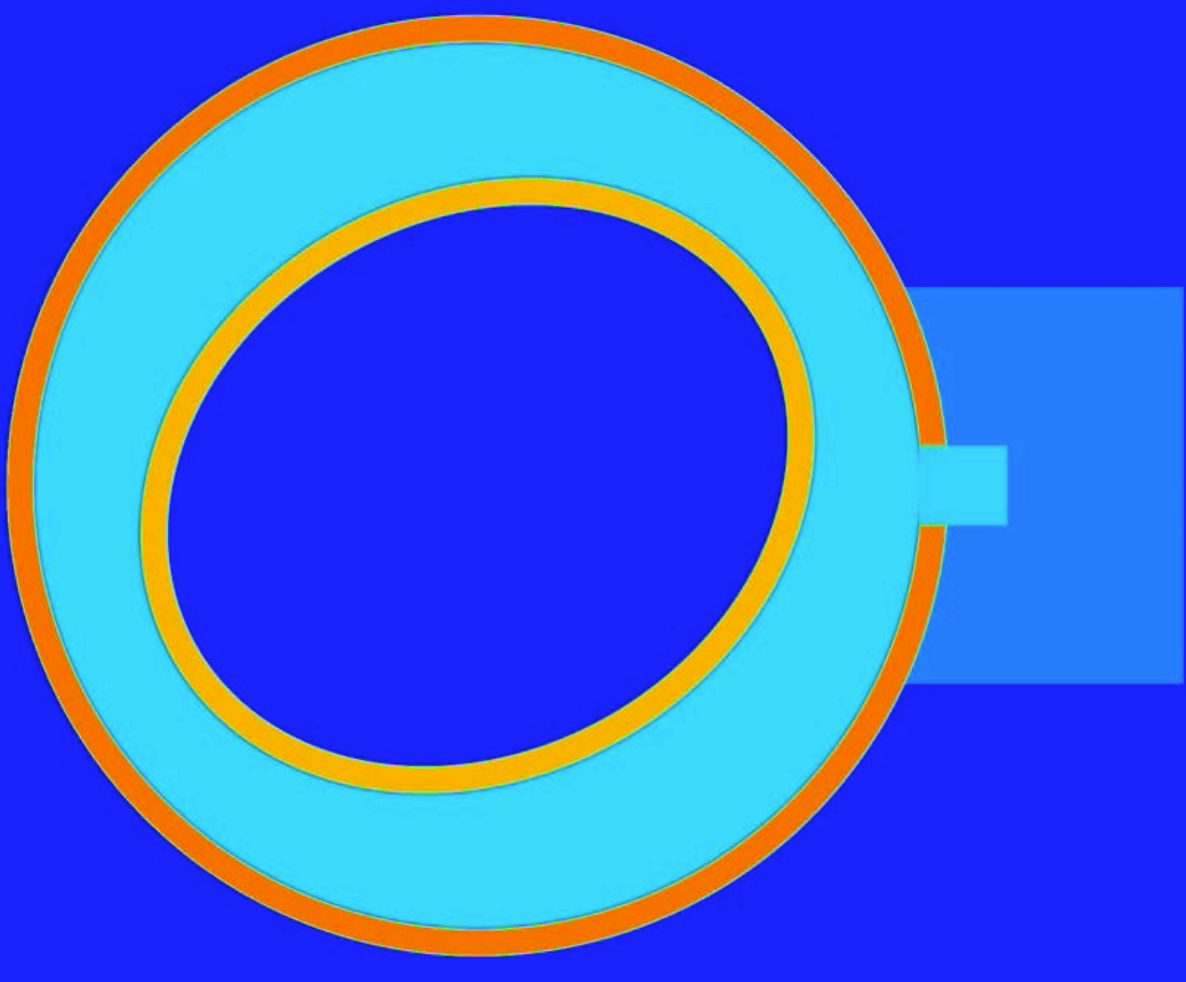




\section{What We See}

- The movie on the preceding slide shows two series of images, the first for the computed density field and the second for the computed gradient of density field overlaid on the PRAD photographs.

- Each series shows the results for the computed solution at a series of resolutions.

- As the resolution increases (finer mesh at the burn front) the velocity of the front increases and converges toward the experimental interface.

-We clearly see the reverberated waves in metal shells that impact the PDV measurements.

- The biggest difference is in the position and shape of the metal shells - Not directly relevant for the HE burn front validation as the difference occur downstream from the burn front

- At later times the steel shell is deflected outward compared to experiment

- The large dent in the tin interface seen in the experiment is noticeably smaller in the computation

- These differences in the metal shell behaviors suggest the SteinbergGuinan strength model is not completely consistent with the real behavior of the metal shells

- Other models might yield improvements 


\section{Quantitative Convergence Validations}

- Quantitative metrics for the convergence of the isochrone burn fronts required fitting the computation and experiment fronts

- The Ensight spline tool proved very useful in fitting the computations.

- The SURF burn model detects the high explosive ignition using the value of the Hugoniot function. This field is printed in the xRage restart dumps and could be used to fit a spline curve to the lead front.

- Isochrone spline fits were computed for each of the six resolution levels and thirty time frames using the Ensight spline tool.

- These fits were further smoothed using smoothing spline tools available from the MATLAB curve fitting package.

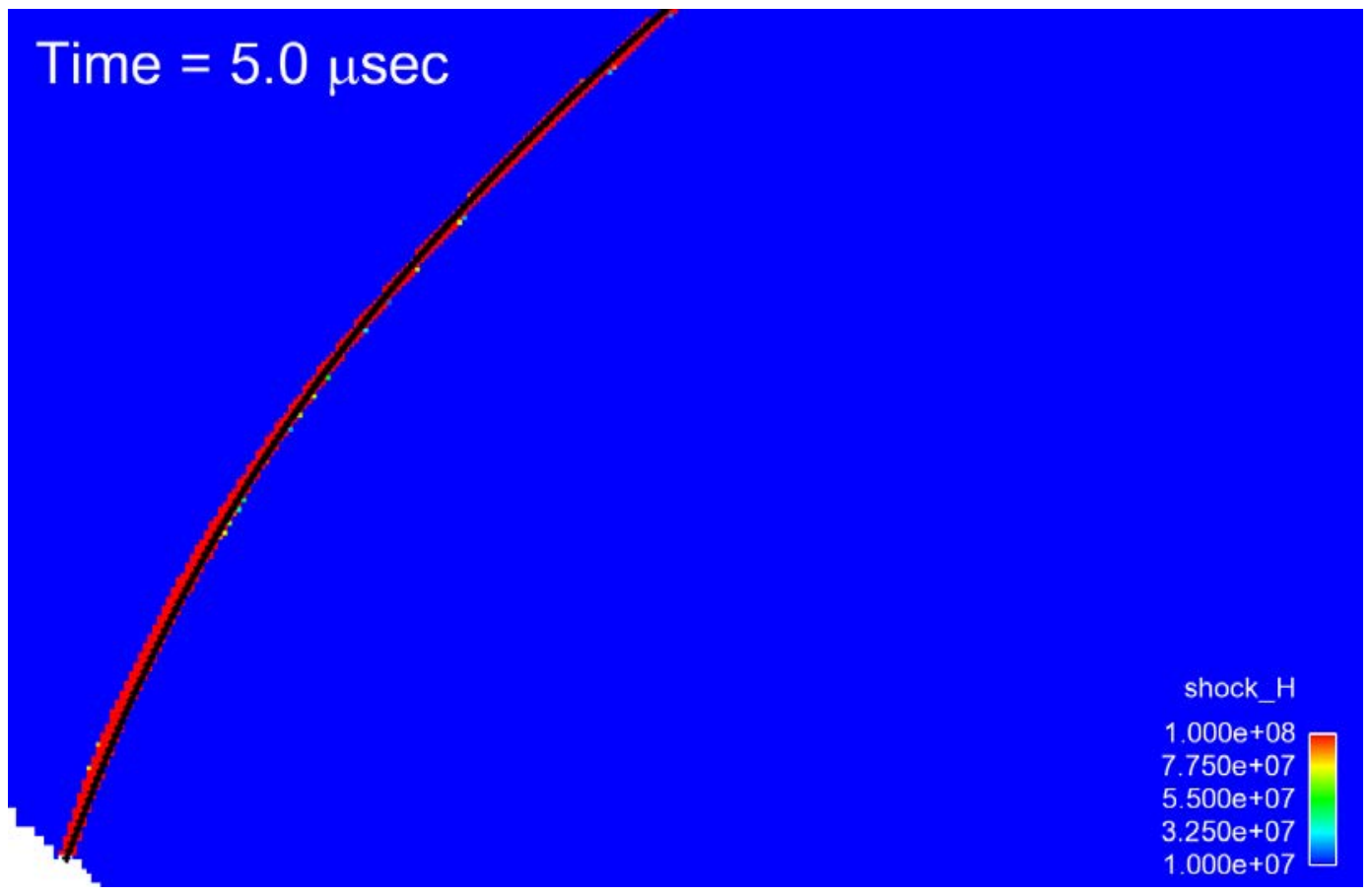




\section{Experimental Isochrone Fits}

- Fitting spline curves to the experimental PRAD images was complicated due to the low resolution of the images.

- Three curves were fit by hand corresponding the leading edge of the burn profile, the middle region, and the trailing edge.

- The difference between the leading and trailing edges gives a rough estimate of the uncertainty in the burn front location.

- The leading edge position is most comparable to the computational fits.

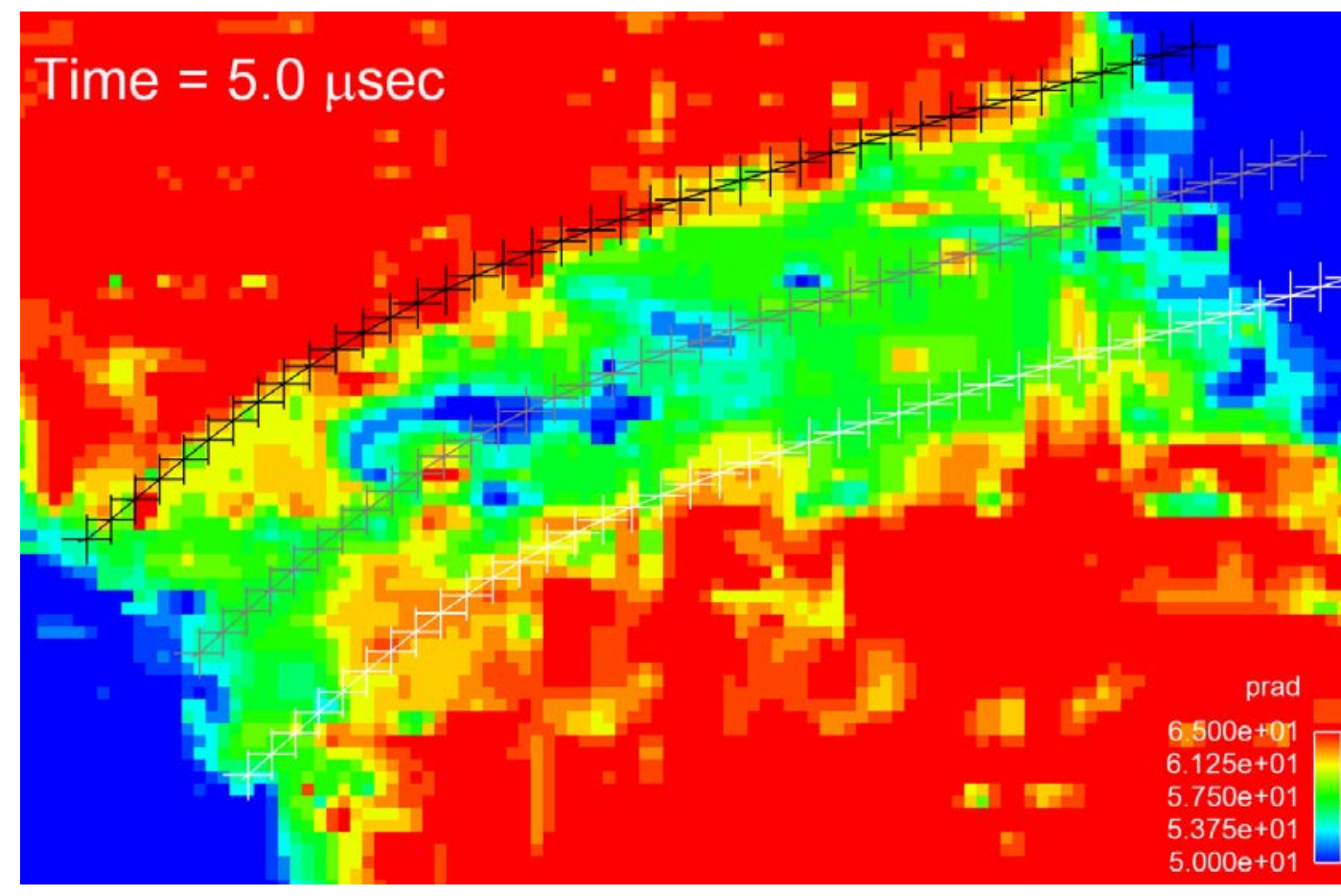




\section{Qualitative Convergence (eye-ball norm)}

- Each of the isochrones are labeled by the time in microseconds when the PRAD image was taken. Each time yields two isochrones, one for the upper section of the device (labeled $U$ ) and one for the lower section (labeled $\mathrm{L}$ ).

\section{Isochron positions}

- These images occur at times ranging from four to eighteen microseconds.

- Beyond eighteen microseconds the upper and lower burn fronts have collided.

- The figure shows the computed and experimental isochrones superimposed.

- With increased refinement the computed values converge towards experiment

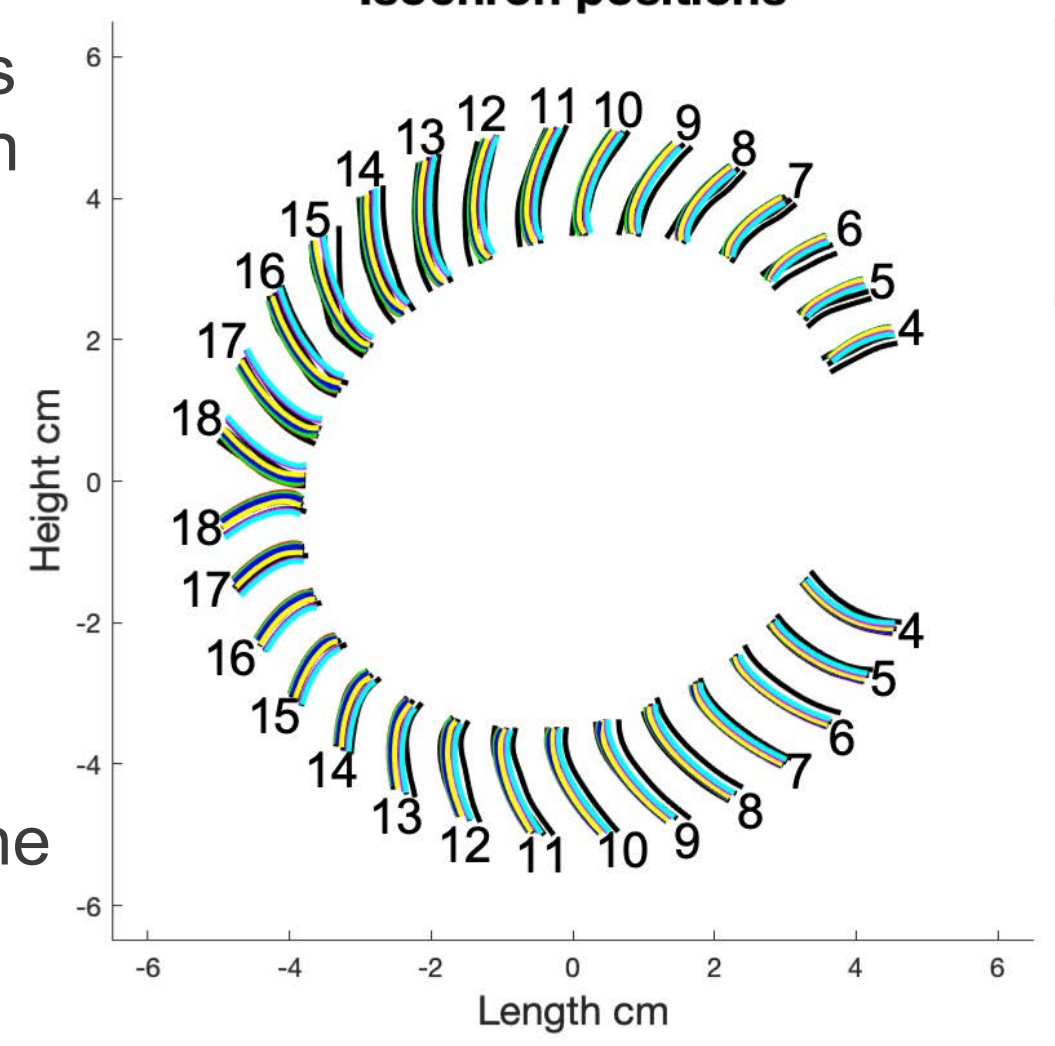




\section{Blow-up of Isochrones at 16 microseconds}

- The picture on the previous slide is a bit difficult to see.

- Below are blow-ups of the 16 microsecond images.
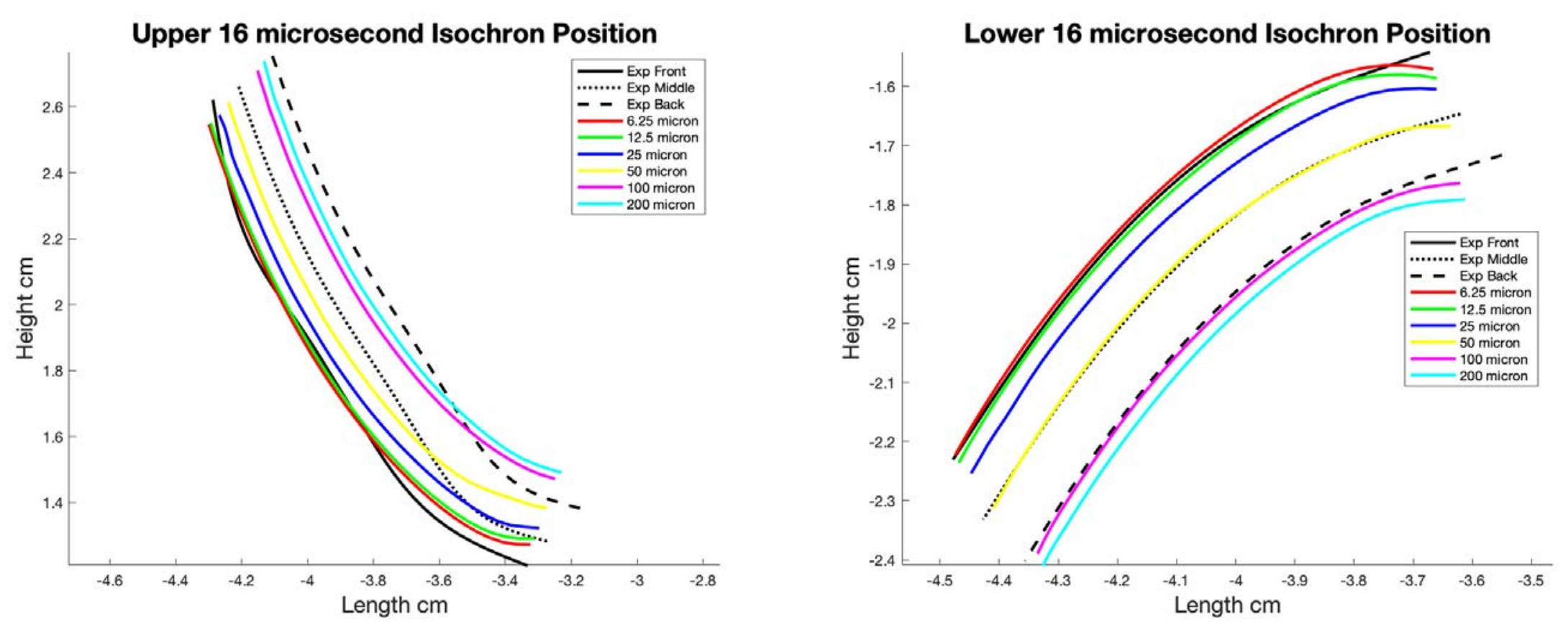

- By the 6.25 micro resolution the computed and picked isochrones agree closely in position

- The resolution of the PRAD photographs was not sufficient to see the curvature at the inner edge of the channel. 


\section{Convergence Rates for Isochrones}

- For each of the isochrones from four to eighteen microseconds we computed the convergence rate to the experimental data using loglinear regression.

- The convergence was computed at a common arc length along each isochrone.

Upper 16 microsecond Isochron Convergence Rate to Experiment Mean $=0.68212$

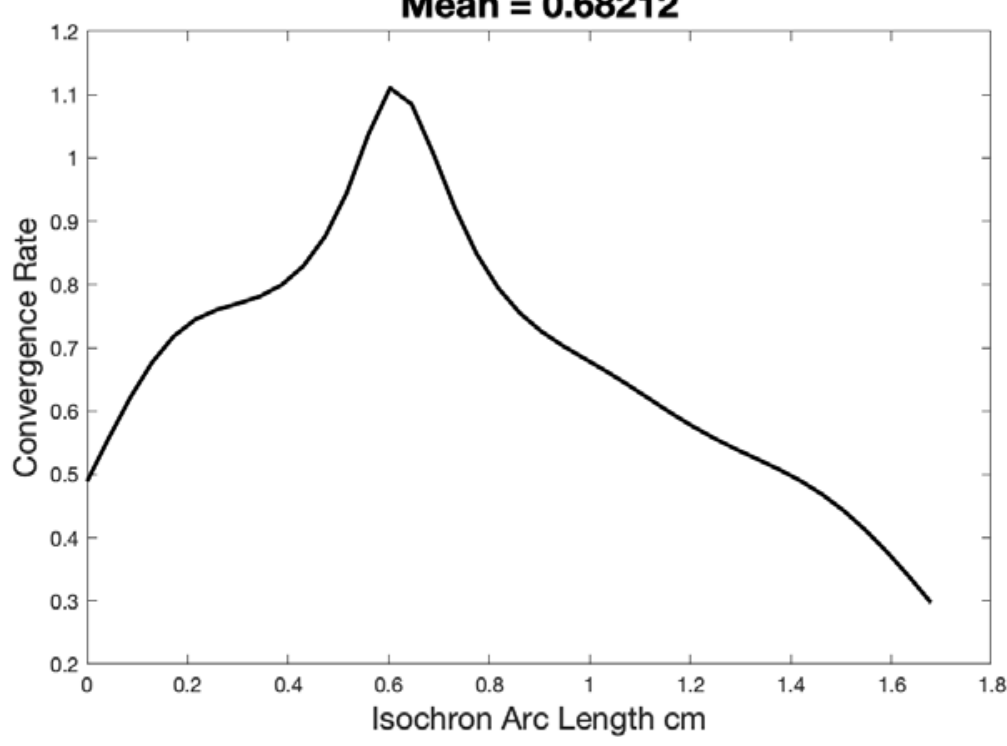

Lower 16 microsecond Isochron Convergence Rate to Experiment Mean $=1.06712$

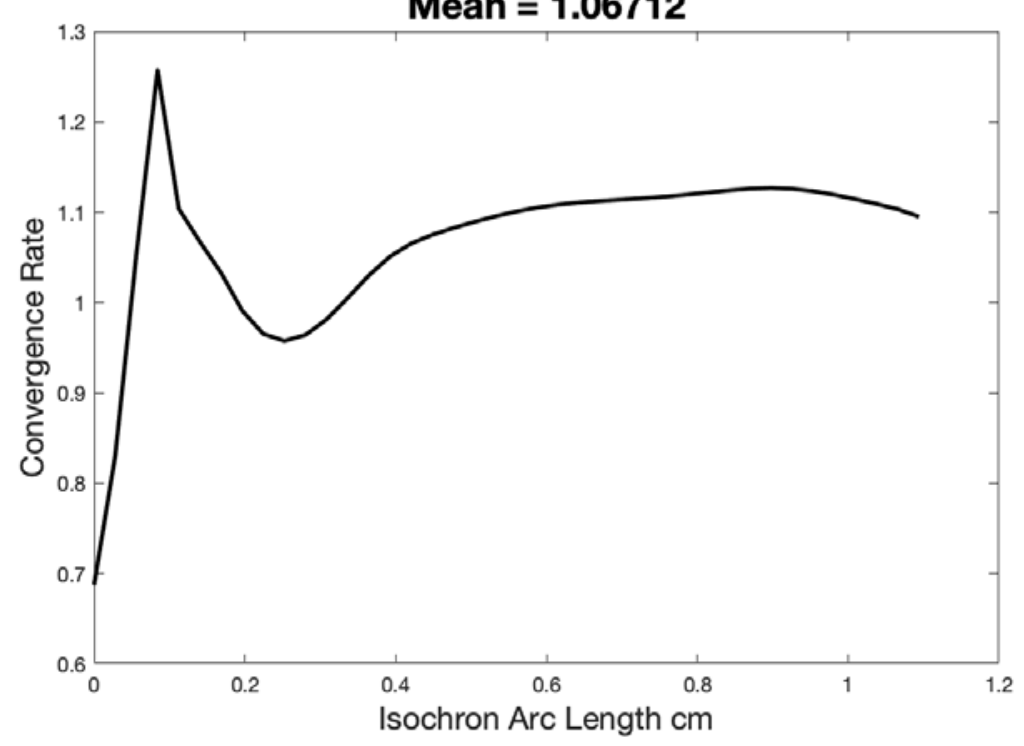




\section{Integrated Convergence Rates}

- If we integrate the convergence rates along each isochrone we get a picture of an average convergence rate.

- The figure shows convergence of the averaged rates of about first order on the 16 microsecond isochrone.

- The metric is the convergence rate of the integrated difference between the computed and picked isochrone positions as a function of arc length.

- The 200 micron case is an outlier. For resolutions finer than 200 the actual rate will be higher

- We see a slight glitch at 6.25 micron resolution on the lower isochrone. At this point the grid size is comparable to the grain size so the continuum assumption may be becoming invalid.

- This feature may also be related to the somewhat subjective fit to the PRAD data.

- Similar rates are seen on all of the isochrones.

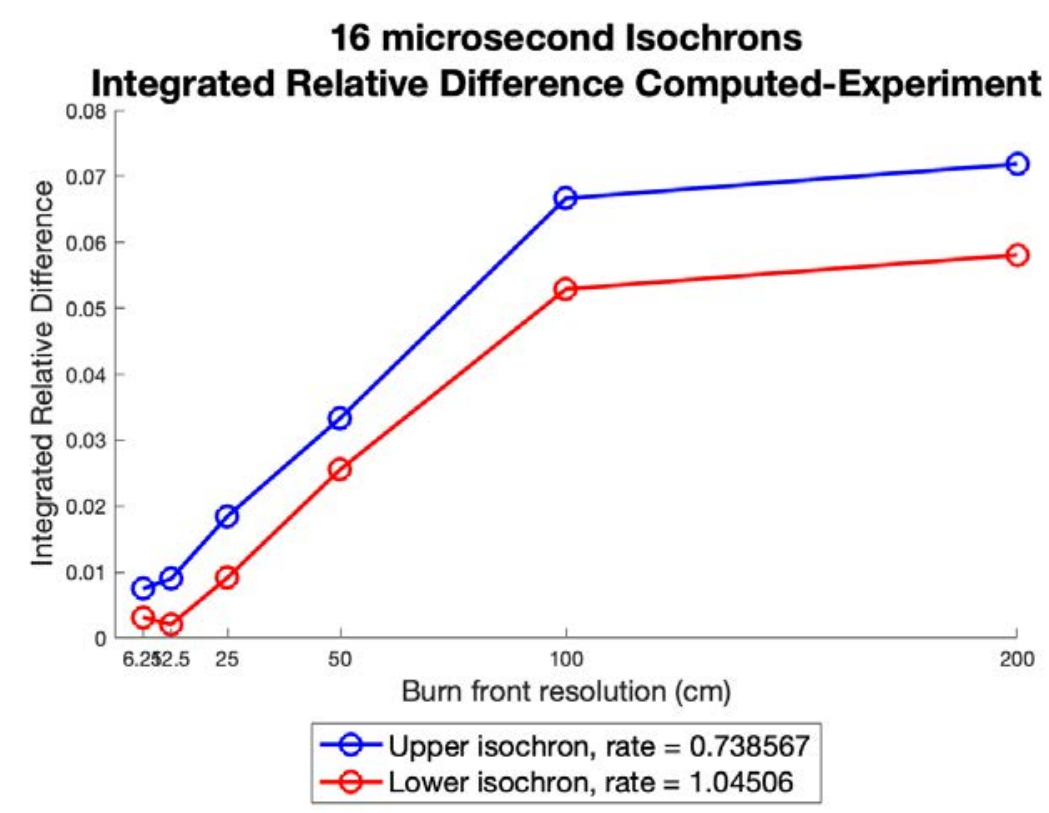




\section{Verification Rates}

- The relatively poor resolution of the experimental PRAD images makes detailed comparisons subject to a substantial "human" error in picking out the locations of the PRAD measured burn fronts.

- We also computed solution verification rates based on the 6.25 micron resolution case as a fiducial.

- In this case we get approximate first order convergence towards the fiducial.

Upper 16 microsecond Isochron

Simulation Convergence Rate

Mean = 1.16045

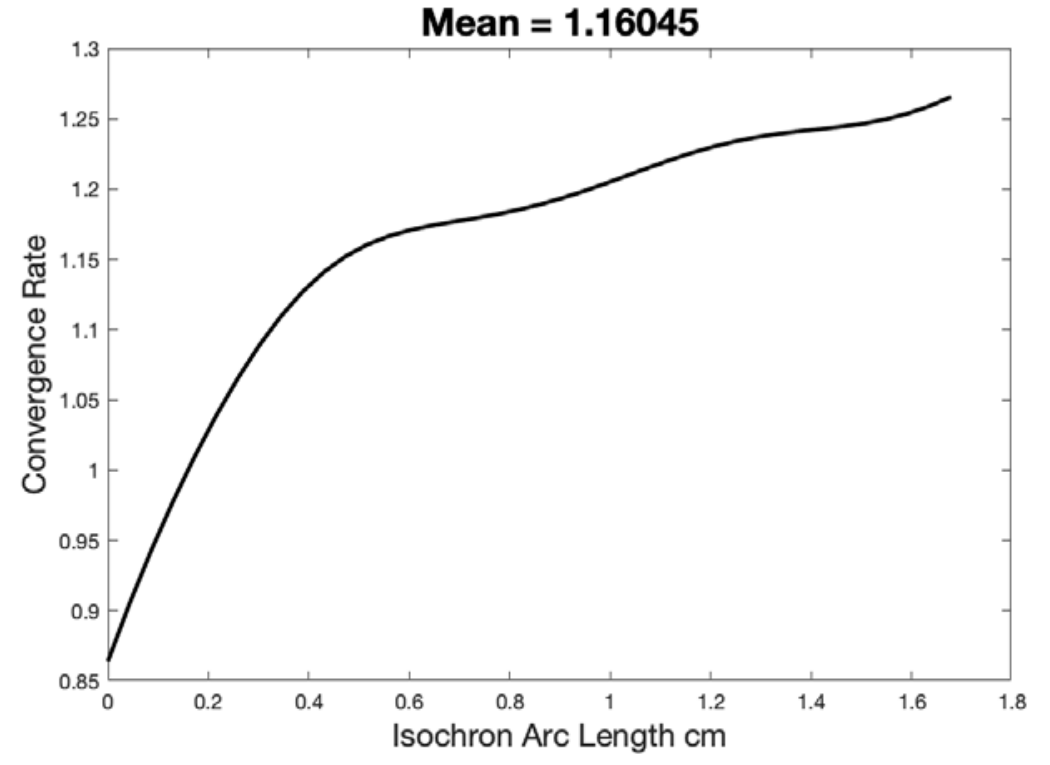

Lower 16 microsecond Isochron Simulation Convergence Rate Mean $=1.03171$

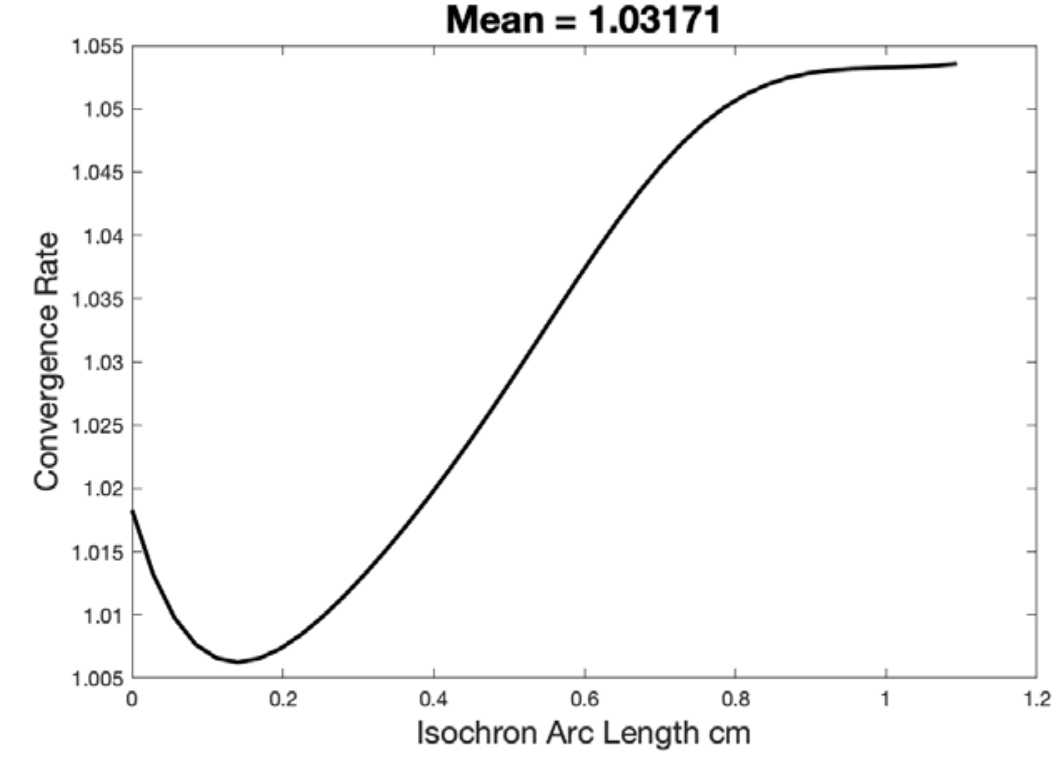




\section{Integrated Verification Rates}

- As with the experimental rates we computed the integrated convergence rates.

- As before this is convergence rate of the integral of the difference between the isochrones as a function of arc length.

- We note the the glitch at the finest resolution seen in the experimental comparison is not apparent.

- Of course the glitch occurred at the finest resolution, which is now take as the fiducial, so the absence of the glitch is perhaps not surprising.

- As before the 200 micron case is an outlier

16 microsecond Isochrons

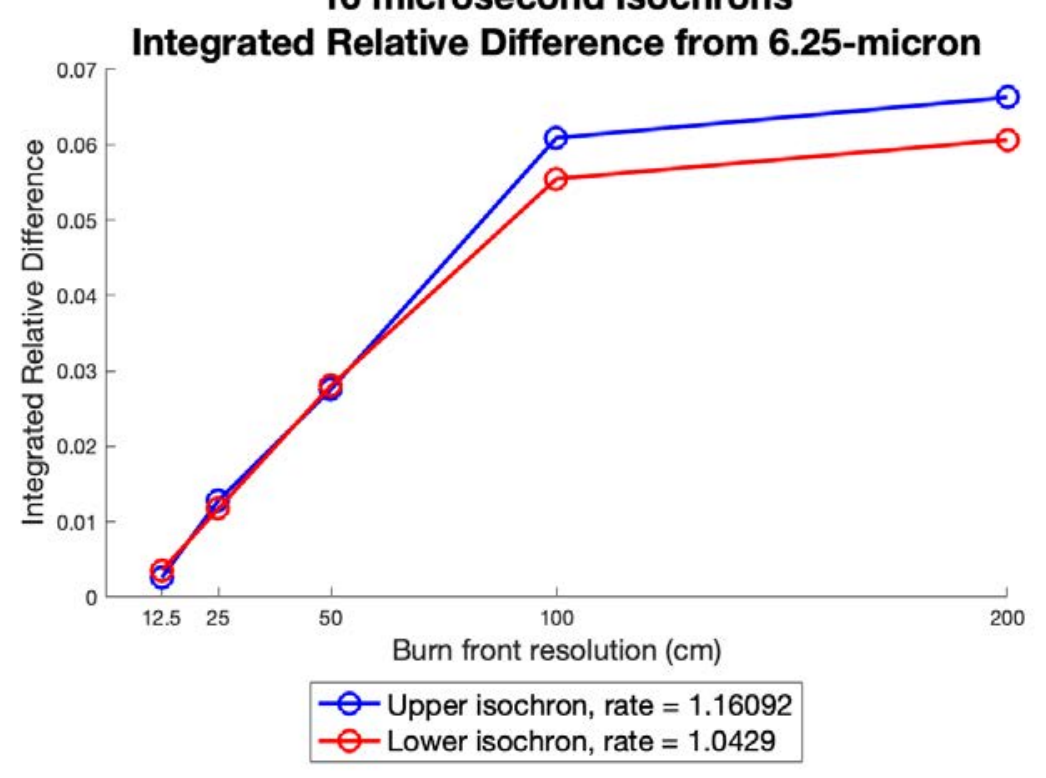




\section{Convergence Rate Summary}

- Lack of space and time prevent showing graphs of the rates for all of the isochrones. The table below gives a summary for all of the isochrones

\begin{tabular}{|c|c|c|c|c|}
\hline $\begin{array}{l}\text { Isochrone Arrival } \\
\text { Time microseconds }\end{array}$ & $\begin{array}{l}\text { Lower Isochrone } \\
\text { Mean Convergence } \\
\text { Rate Computation to } \\
\text { Experiment }\end{array}$ & $\begin{array}{l}\text { Upper Isochrone } \\
\text { Mean Convergence } \\
\text { Rate Computation to } \\
\text { Experiment }\end{array}$ & $\begin{array}{l}\text { Lower Isochrone } \\
\text { Mean Convergence } \\
\text { Rate Computation to } \\
\text { Fiducial }\end{array}$ & $\begin{array}{c}\text { Upper Isochrone } \\
\text { Mean Convergence } \\
\text { Rate Computation to } \\
\text { Fiducial }\end{array}$ \\
\hline 4 & 0.707116 & 0.186394 & 1.10166 & 1.78911 \\
\hline 5 & 0.744946 & 0.287773 & 1.15774 & 1.39983 \\
\hline 6 & 0.950369 & 0.34758 & 1.19751 & 1.49275 \\
\hline 7 & 0.37414 & 0.380441 & 1.19203 & 1.34514 \\
\hline 8 & 0.393144 & 0.406448 & 1.13889 & 1.28526 \\
\hline 9 & 0.801305 & 0.402676 & 1.15045 & 1.27982 \\
\hline 10 & 0.654035 & 0.498127 & 1.12111 & 1.15437 \\
\hline 11 & 0.548552 & 0.511603 & 1.13857 & 1.25483 \\
\hline 12 & 0.566443 & 0.435021 & 1.06179 & 1.16364 \\
\hline 13 & 0.650162 & 0.548418 & 1.1099 & 1.26979 \\
\hline 14 & 0.84343 & 0.50915 & 1.05048 & 1.19117 \\
\hline 15 & 0.770116 & 0.507175 & 1.02866 & 1.2005 \\
\hline 16 & 1.04506 & 0.738567 & 1.0429 & 1.16092 \\
\hline 17 & 0.652512 & 0.629139 & 1.00663 & 1.11089 \\
\hline 18 & 0.56787 & 0.753479 & 0.960375 & 1.04437 \\
\hline
\end{tabular}




\section{Average Rate Over All Isochrones}

- As a final convergence metric we computed the average over all isochrones for the positional convergence, both to experiment and to the 6.25 micron fiducial.

- Convergence towards experiment is about $1 / 2$ order and about first order towards the fiducial
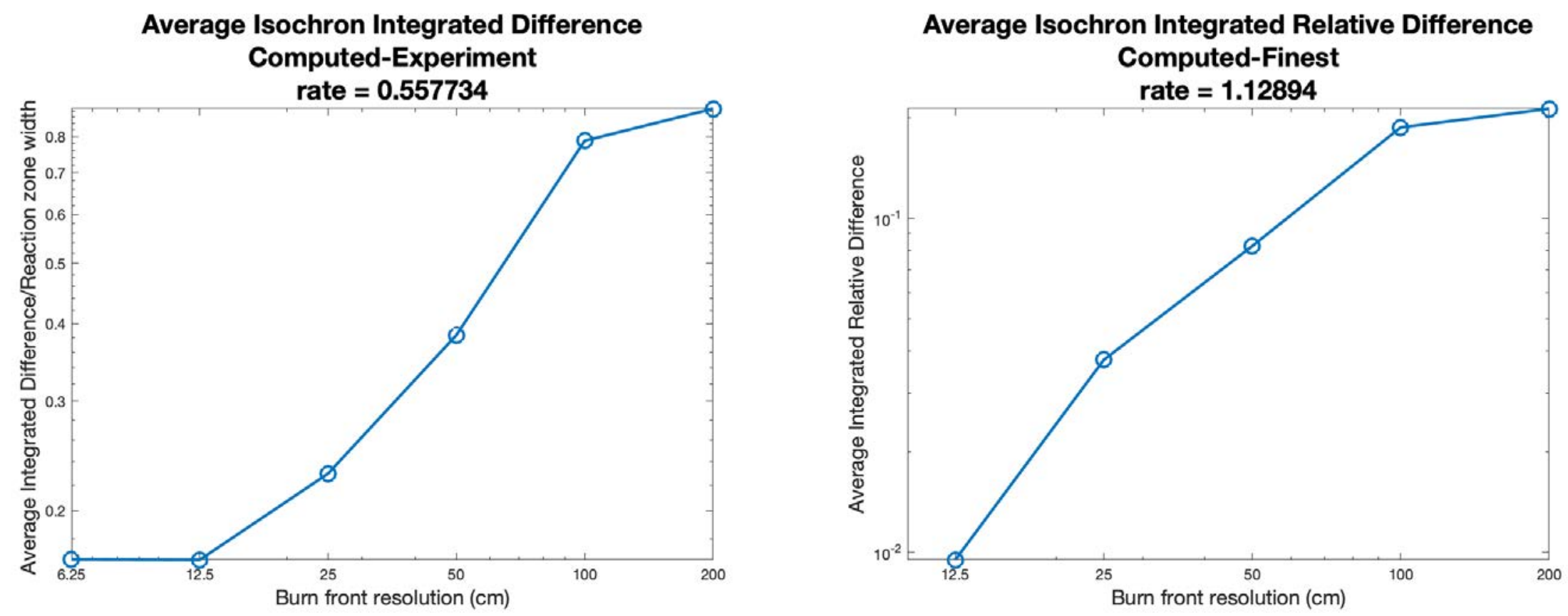


\section{Burn Front Curvature}

- One of the features of the SURFplus model is to incorporate the geometric source terms corresponding to burn front curvature into the SURFplus fitting forms.

- We test the success of these fits by examining the burn front curvature compared both the experiment and the simulation fiducial.

- The two figures show the gradient of density superimposed over the PRAD images for the 50 micron and 6.25 micron cases.
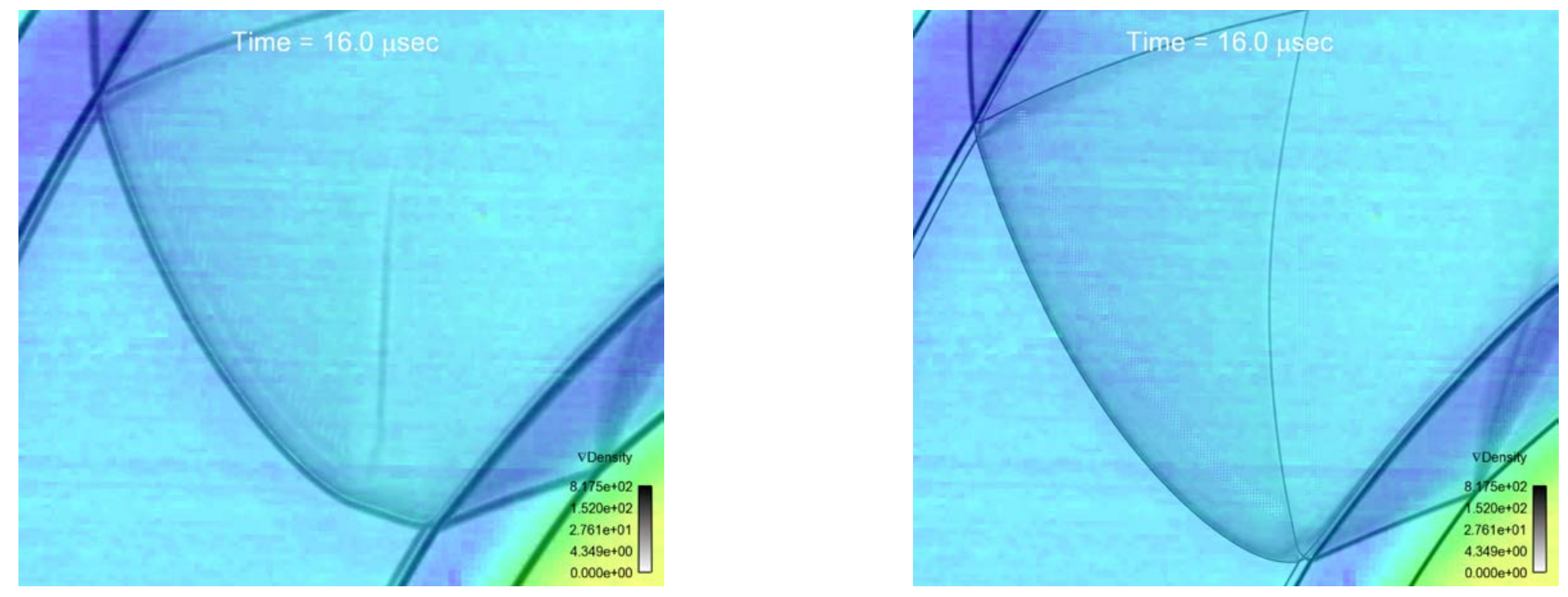


\section{Experimental Curvature Comparison}

- The somewhat "ad hoc" fitting for the PRAD isochrones is subject to considerable variation in the fitted front curvature

- The figures show the computed verses experiment fitted front curvatures.

- The computations are indicating a convergence towards the finest resolution, but the experiment fits are all over the place

Upper 16 microsecond Isochron Simulation Curvature Convergence Rate

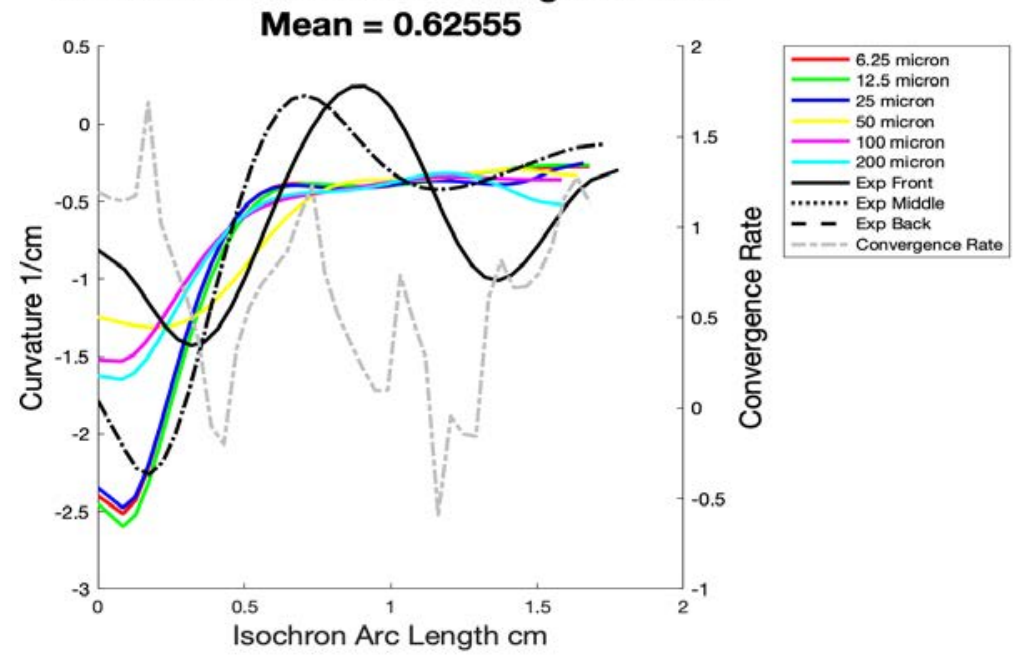

Lower 16 microsecond Isochron Simulation Curvature Convergence Rate

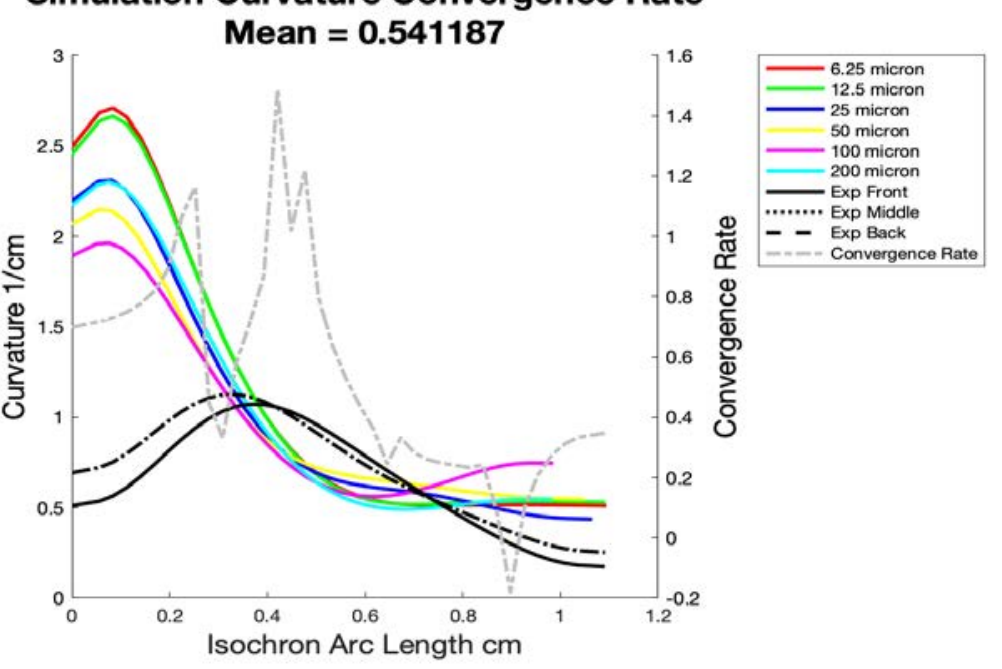




\section{Curvature Verification}

- The noisy resolution at the fine scale needed to fit the experiment led us to focus instead on a verification of the curvature convergence rather than an explicit validation to the experiment.

- As before we use the example of the 16 microsecond isochrones compared to the 6.25 fiducial.

- Comparing the integrated difference of the curvatures for each resolution to the 6.25 micron fiducial we see about $3 / 4$ accurate convergence behavior

- The average over all isochrones shows about a half order accurate convergence
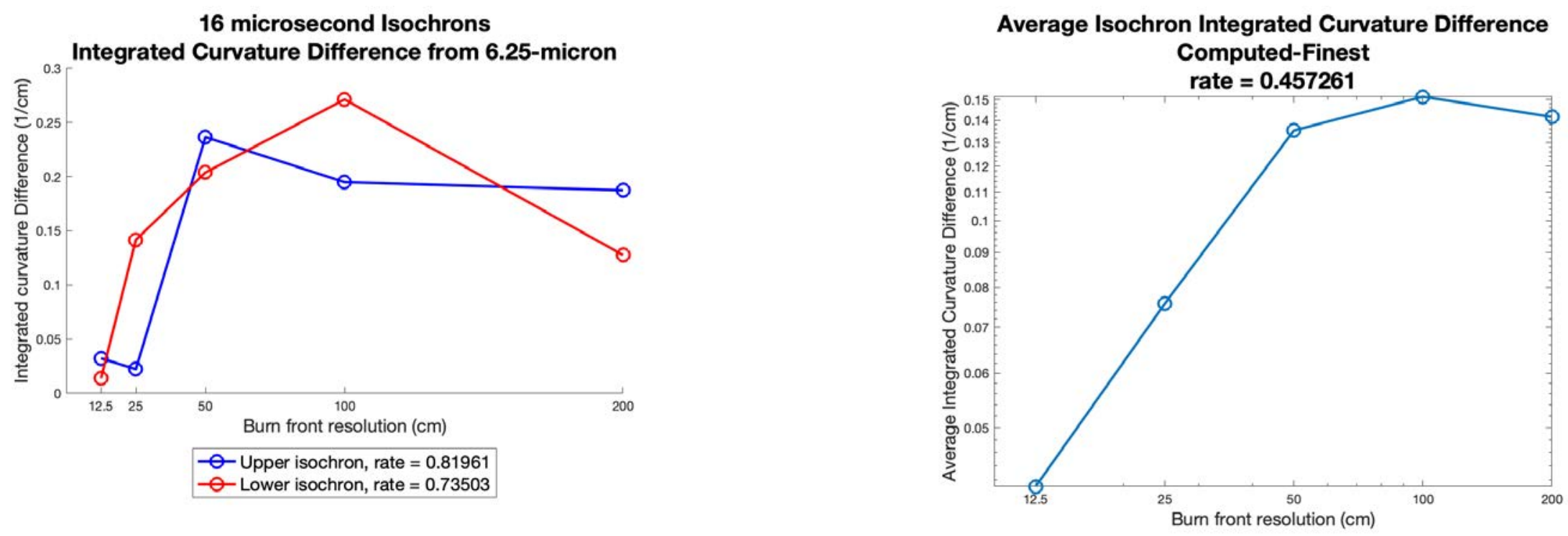


\section{Sobolev Convergence}

- As a final converge test we compute the $W^{2,1}$ norm for the difference of the solutions compared to the 6.25 micron fiducial, where the norm for the Sobolev space space $W^{k, p}$ is defined as

$$
\|f\|_{k, p}=\left(\sum_{i=1}^{k}\left\|f^{(i)}\right\|_{p}^{p}\right)^{1 / p}=\left(\sum_{i=1}^{k} \int\left|f^{(i)}(t)\right|^{p} d t\right)^{1 / p}
$$

- The figures indicate that for this norm we are getting close to first order converged in the combined position and shape for the burn front geometry compared to the 6.25 micron fiducial

- We note that this norm is dominated by the position differences
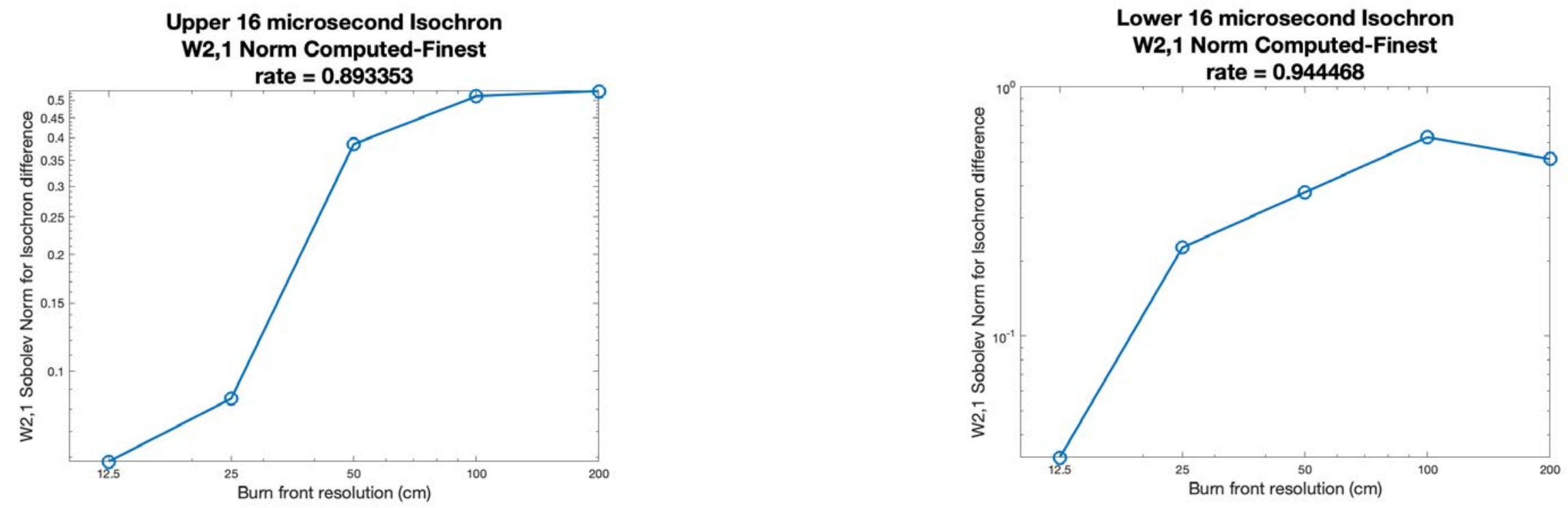


\section{Conclusions}

- We discussed a variety of convergence metrics for the validation of the xRage simulations of the Cyclops I experiment based on two diagnostics, photon-Doppler-velocimetry and proton-radiography images.

- The simulations show the expected convergence properties under mesh refinement of the reaction front resolution from 200 down to 6.25 microns.

- The PDV probe comparisons agreed with experiment for the time of arrival of the high explosive generated shock wave at the outer edge of the steel shell to within a couple of percent and the finer resolutions agreed with experiment at the $1 \%$ or better level.

- The average for the spline fit isochrones showed convergence to the experimental fit isochrones to better than a half order of accuracy and the computed positions agreed with experiment to better than $5 \%$ for resolutions less than 50 microns and no worse than about $10 \%$ for all resolutions. At the finest level of refinement, the agreement with the isochrone positions was under $1 \%$. 


\section{Conclusions}

- We also discussed verification metrics to illustrate that selfconsistency of the xRage code and its implementation of the SURFplus model under mesh refinement.

- These metrics show that SURFplus combined with the xRage hydrodynamics method achieve on average a better than first order convergence to the finest grid simulation taken as the fiducial.

- Since these positions are all related to the lead shock front, first order convergence is the best that could be expected for a conservative Godunov algorithm.

- Based on the above metrics both in terms of agreement with experiment and solution verification we can have good confidence with the simulation results using the SURFplus high explosive model for all resolutions finer than 50 microns (about two mesh zones in the reaction profile) and that even at coarser resolutions the calculations agree with experiment to within $10 \%$. 